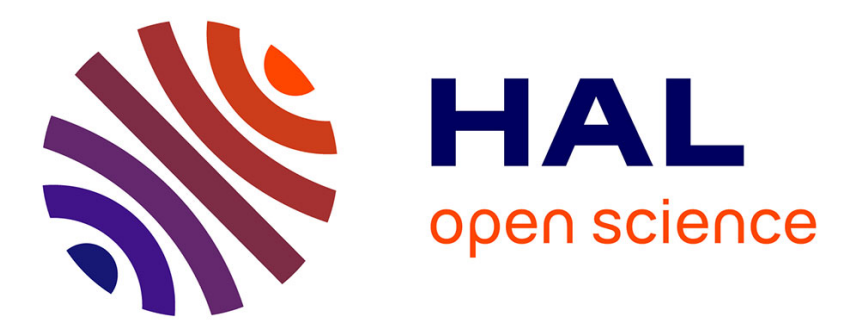

\title{
Benchmarking of oxygen evolution catalysts on porous nickel supports
}

Adèle Peugeot, Charles E Creissen, Dilan Karapinar, Moritz Schreiber, Marc

Fontecave, Ngoc Tran Huan

\section{To cite this version:}

Adèle Peugeot, Charles E Creissen, Dilan Karapinar, Moritz Schreiber, Marc Fontecave, et al.. Benchmarking of oxygen evolution catalysts on porous nickel supports. Joule, 2021, 5 (5), pp.1281-1300. 10.1016/j.joule.2021.03.022 . hal-03253478

\section{HAL Id: hal-03253478 https://hal.sorbonne-universite.fr/hal-03253478}

Submitted on 8 Jun 2021

HAL is a multi-disciplinary open access archive for the deposit and dissemination of scientific research documents, whether they are published or not. The documents may come from teaching and research institutions in France or abroad, or from public or private research centers.
L'archive ouverte pluridisciplinaire HAL, est destinée au dépôt et à la diffusion de documents scientifiques de niveau recherche, publiés ou non, émanant des établissements d'enseignement et de recherche français ou étrangers, des laboratoires publics ou privés. 
Adèle Peugeot ${ }^{a}$, Charles E. Creissen ${ }^{a}$, Dilan Karapinar ${ }^{a}$, Huan Ngoc Tran ${ }^{a}$, Moritz Schreiber ${ }^{b}$ and Marc Fontecave $^{\mathrm{a} *}$

a Laboratoire de Chimie des Processus Biologiques, CNRS UMR 8229, Collège de France, Sorbonne Université, Paris, France

${ }^{\mathrm{b}}$ Total Research and Technology, Refining and Chemicals, Division $\mathrm{CO}_{2}$ Conversion, Feluy, 7181 Seneffe, Belgium

*correspondence to: marc.fontecave@college-de-france.fr

\section{Context \& Scale}

9

Storage of intermittent renewable energy can be achieved through the conversion of electricity into chemical energy. The solar- and electricity-driven production of energy vectors such as hydrogen, alcohols and hydrocarbons can be achieved in electrochemical cells, where proton and $\mathrm{CO}_{2}$ reduction at the cathode is coupled to water oxidation at the anode. To develop these processes for high-rate, energy efficient experimental demonstrations, highly active and stable oxygen evolution reaction (OER) catalysts are needed. The present benchmarking study, using a standardised characterisation protocol, allows for rigorous identification of the current best solid-state OER catalysts based on non-precious metals immobilised on a Ni foam support. Further optimisation of anodes will emerge not only through tuning the composition and morphology of the catalysts but also from the design and integration of novel conductive and structured supports. 
Active and inexpensive oxygen evolution reaction (OER) electrocatalysts are required for energy efficient electrolysis applications. Objective comparison between OER catalysts has been blurred by the use of different supports and methods to evaluate performance. Here, we selected nine highly active transitionmetal-based catalysts and described their synthesis, using a porous nickel foam and a new Ni-based dendritic material as the supports. We designed a standardised protocol to characterise and compare the catalysts in terms of structure, activity, density of active sites and stability. NiFeSe and CoFeSe derived oxides showed the highest activities on our dendritic support, with low overpotentials of $\eta_{100} \approx 247 \mathrm{mV}$ at $100 \mathrm{~mA} \mathrm{~cm}^{-2}$ in $1 \mathrm{M} \mathrm{KOH}$. Stability evaluation showed no surface leaching during 8 hours electrolysis. This work highlights the most active anode materials and provides an easy way to increase the geometric current density of a catalyst by tuning the porosity of its support.

Keywords Metal Oxides 
The oxygen evolution reaction (OER) is one of the most relevant anodic reactions within electrochemical cells, where it is coupled to the hydrogen evolution reaction $(\mathrm{HER})^{1-6}$ or the $\mathrm{CO}_{2}$ reduction reaction $\left(\mathrm{CO}_{2} \mathrm{RR}\right)$ to energy-dense carbon compounds at the cathode..$^{7-9}$ It is thus of high relevance for electrochemical energy conversion and storage technologies. Oxygen generation from water oxidation at the anode is typically carried out in acidic or alkaline conditions. Operation in alkaline conditions allows the use of cheap, efficient and stable non-precious-metal catalysts, in contrast to acidic conditions, in which only expensive and scarce noble metal based catalysts such as $\mathrm{IrO}_{2}$ and $\mathrm{RuO}_{2}$ exhibit significant stability. ${ }^{10}$ In alkaline conditions, the best performance and highest stabilities were observed for Ni based multimetallic catalysts, ${ }^{11-15}$ which led to their widespread use as OER catalysts. ${ }^{10,16-19}$ However, sluggish kinetics of the four electron oxygen evolution reaction requires a significant anodic overpotential to achieve relevant geometric current densities, reducing the efficiency of the conversion of electrical to chemical energy. Hence identifying efficient, cheap and stable OER catalysts comprised of Earth-abundant elements is of fundamental importance and has been a prominent field of research during the last 20 years. $^{20-23}$ Among non-noble multimetallic metal-based OER catalysts reported so far, mixed nickel/iron/cobalt oxides in particular have shown stable low overpotentials at relevant geometric current densities.

Benchmarking these novel OER electrocatalysts is of utmost importance but remains highly challenging, since methods for evaluating performance (activity and stability) are non-standardised, making fair and reliable comparison extremely difficult. Notably, different catalyst supports are used, to which OER is very sensitive, ${ }^{24,25}$ and variation in characterisation methods and experimental setups further adds to disparities between reported performances. As a matter of fact, very few benchmarking studies have been carried out so far. The last significant efforts in providing meaningful benchmarking studies were carried out in $2012,{ }^{26} 2013^{27}$ and $2015 .{ }^{28}$ These studies, conducted on OER electrocatalysts deposited on 
$\mathrm{Au} / \mathrm{Ti}$ or glassy carbon electrodes, performed water oxidation at a current density of $10 \mathrm{~mA} \mathrm{~cm}{ }^{-2}$ with observed overpotentials higher than $300 \mathrm{mV}$. A relevant figure of merit from these studies is the overpotential (denoted as $\eta_{10}$ ) required to achieve $10 \mathrm{~mA} \mathrm{~cm}^{-2}$ current density per geometric surface area at ambient temperature and $1 \mathrm{~atm} \mathrm{O}_{2}$. The $\eta_{10}$ value is indeed the benchmarking criterion generally used in literature. ${ }^{26-28}$ Some OER electrocatalysts reported during the last five years exhibit $\eta_{10}$ overpotentials much closer to $200 \mathrm{mV}$, representing a significant advancement in the field. We thus found timely to provide a fair comparison of the performances of the most active catalysts of this new generation.

Here, we establish a protocol to benchmark a range of anodes, comprised of various catalysts synthesised on the same nickel foam (NF) support. Due to its conductivity, mechanical strength, relative inertness at alkaline $\mathrm{pH}$, and low cost, nickel is an efficient current collector and a good support for active material deposition. Furthermore, nickel foam shows extended geometric surface areas and fine threedimensional structures which make it attractive as a support for heterogeneous catalysts. ${ }^{25,29}$ Although various porous metallic foams have been used in the past for water oxidation, ${ }^{30-32}$ a recent resurgence in the usage of NF as a support material has occurred. This was partly driven by the development of energystorage electrochemical systems in alkaline conditions, such as solar-driven water splitting and electrocatalytic $\mathrm{CO}_{2} \mathrm{RR}$ using gas-fed flow cells. We have followed this trend and used NF as the catalyst support exclusively. ${ }^{17-19}$

For this study, we selected nine promising multimetallic, non-precious-metal catalysts reported in the literature (Table 1). Several selection criteria were adopted such as the $\eta_{10}$ overpotential in alkaline conditions, the Tafel slope, the maximum current density and the long-term stability, as these data were available in the publications. ${ }^{33-41}$ We also made sure to select materials composed of a variety of transition metals, with different morphologies and synthetic procedures, in order to broaden the scope of our comparison. These catalysts were preferentially chosen on the basis that they display low $\eta_{10}$ overpotentials (below $300 \mathrm{mV}$ ). However, we also included the Co-based catalyst developed by Nocera et. 
al. $(\mathrm{CoPi}),{ }^{34}$ despite it exhibiting a $\eta_{10}$ value greater than $300 \mathrm{mV}$ in $1 \mathrm{M} \mathrm{KOH}$, since it is widely used in literature. The NiMoFe-O catalyst was included as it was reported to be the highest performing catalyst at the time of writing. ${ }^{27,42} \mathrm{CoV}-\mathrm{O}$ and $\mathrm{CoV}-\mathrm{OOH}$ were chosen to provide a comparison between two catalysts based on cobalt and vanadium that were synthesised using different methods. In the publications, these catalysts were characterised under very different conditions. In particular, various electrode supports were used (glassy carbon, $\mathrm{Cu}$ plate or $\mathrm{Ni}$ foam), illustrating the difficulty of comparing them just on the basis of the literature data. In our study, we exclusively used the same $\mathrm{Ni}$ foam as the conductive support. The syntheses followed the reported procedures as strictly as possible with slight adaptations to enable deposition on a $1 \mathrm{~cm}^{2} \mathrm{NF}$ support.

\begin{tabular}{ccc} 
Catalyst & Synthesis procedure & Ref \\
\hline Cu-O & Electrodeposition & 33 \\
CoPi & Electrodeposition & 34 \\
NiMoFe-O & Electrodeposition & 35 \\
NiFe-OOH & Precipitation & 36 \\
NiFeSe-dO & Hydrothermal & 37 \\
CoFeSe-dO & Hydrothermal & 38 \\
FeCoW & Gel formation & 39 \\
CoV-OOH & Coprecipitation & 40 \\
CoV-O & Hydrothermal & 41
\end{tabular}

Table 1 Oxygen Evolution catalysts with their method for synthesis used in this study

The materials were characterised and their kinetic performances for the OER in alkaline conditions analysed. Geometric current densities of OER catalysts must attain several hundreds of milliamperes per square centimetre to facilitate $\mathrm{CO}_{2}$ reduction and solar-driven water splitting under lab-scale conditions. ${ }^{1,2,43}$ Therefore, we benchmarked the catalysts at the commonly reported value of $10 \mathrm{~mA} \mathrm{~cm}^{-2}$ but also at a more relevant current density of $100 \mathrm{~mA} \mathrm{~cm}^{-2},{ }^{17,44-47}$ according to a standardised protocol. Catalysts must be compared in terms of intrinsic activities, which requires to normalise the current densities by the effective electrochemical surface areas (ECSAs). This is in general a very challenging issue, quite often incompletely addressed. We have thus been careful in providing an array of experimental 

procedures for determination of concentrations of active sites and specific activities, and we propose a best-practice protocol for researchers in the field. Finally, we report a novel NF-based support with different morphology and increased structuration, leading to significant improvement of the performances

4 of almost all studied catalysts. By doing so, we report two OER catalysts with overpotential values of 195

$5 \mathrm{mV}$ and $198 \mathrm{mV}$ required for a current density of $10 \mathrm{~mA} \mathrm{~cm}{ }^{-2}$, and overpotentials of $247 \mathrm{mV}$ required for

$6100 \mathrm{~mA} \mathrm{~cm}^{-2}$, among the lowest values reported so far. We demonstrate the outstanding electrochemical

7 and compositional stability of these two catalysts, evaluated by a long-term flow experiment.

8 Furthermore, we stress the importance and necessity of performing galvanostatic tests as well as surface

9 leaching quantification in order to assess the stability of the catalyst. 


\section{Experimental Procedures}

Materials Chemical reagents were purchased in reagent grade from Alfa Aesar and Merck. Nickel foam (1.6 $\mathrm{mm}$ in thickness, purity $99.5 \%$, density $0.45 \mathrm{~g} \mathrm{~cm}^{-3}, 95 \%$ porosity, 20 pores $\mathrm{cm}^{-2}$ ) was purchased from Goodfellow. Oxygen 5.0 was purchased from Linde. All electrochemical experiments were performed with a VSP300 BioLogic Potentiostat and the Biologic EC-Lab software was used for data analysis. Hydrothermal syntheses were performed in a Carbolite Gero CWF1213 furnace. SEM images were collected on a SU-70 Hitachi FEGSEM equipped with a X-Max $50 \mathrm{~mm}^{2}$ Oxford spectrometer for energy dispersive X-Ray spectroscopy (EDX) measurements. Inductively coupled plasma - optical emission spectrometry (ICP-OES) measurements were performed on a Thermo Scientific iCAP 6300 duo device. Inductively coupled plasma - mass spectroscopy (ICP-MS) measurements were performed on a ICP-QMS 7900 Agilent apparatus.

Catalyst synthesis Nickel foam (NF) was used as the support for all the catalysts. $1 \mathrm{~cm}^{2}$ square foams were cut, and an additional section was left for electrical contact. This area was partially covered with epoxy glue in order to delimit the $1 \mathrm{~cm}^{2}$ area as precisely as possible. These foams were pre-treated by soaking in a $3 \mathrm{M} \mathrm{HCl}$ solution for 10 minutes, in order to remove the nickel oxide layer formed at the 
Electrochemical characterisation A two-compartment cell separated by a glass frit was used for electrochemical measurements. The electrolyte was an aqueous solution of $1 \mathrm{M} \mathrm{KOH}$. A threeelectrode arrangement using a platinum mesh counter electrode (Goodfellow, $2.25 \mathrm{~cm}^{2}$ ) and a $\mathrm{Ag} / \mathrm{AgCl} / \mathrm{KCl}_{\text {sat }}$ reference electrode (BioLogic), which was very regularly calibrated against potassium ferrocyanide in order to ensure the absence of any shift in its potential. The potentials were reported vs RHE according to the following equation: $E_{\mathrm{RHE}}=\mathrm{E}_{\mathrm{Ag} / \mathrm{AgCl}}+0.197+0.059 \cdot \mathrm{pH}$. The working electrode was positioned in the cell in order to minimize the distance to the reference electrode $(\approx 1 \mathrm{~mm})$, thus avoiding a large contribution from the cell in the ohmic drop (the resistance was always between 0.1 and 0.25 $\Omega)$. Before each set of experiments, $\mathrm{O}_{2}$ was flowed through the working electrode's compartment for 20-30 minutes. This is an important step as it prevents any contribution from the $\mathrm{O}_{2}$ partial pressure $\mathrm{p}_{\mathrm{O}_{2}}$ in the thermodynamic potential calculation: $\mathrm{E}_{\mathrm{Nernst}}=\mathrm{E}_{\mathrm{H}_{2} \mathrm{O} / \mathrm{O}_{2}}-0.059 \cdot \log \mathrm{pH}+0.015 \cdot \log \mathrm{P}_{\mathrm{O}_{2}} \cdot \operatorname{In}$ an $\mathrm{O}_{2^{-}}$ saturated solution $\mathrm{p}_{\mathrm{O}_{2}}=1$, and the thermodynamic potential is given by the following equation: $\mathrm{E}_{\mathrm{Nernst}}$ $=\mathrm{E}_{\mathrm{H}_{2} \mathrm{O} / \mathrm{O}_{2}}-0.059 \cdot \log \mathrm{pH}$.

Each material was characterised in $10 \mathrm{~mL}$ of $1 \mathrm{M} \mathrm{KOH}$ aqueous solution following a precise protocol divided into 3 steps. Step 1 : Consecutive linear sweep voltammetry (LSV) scans were performed at a scan rate of $10 \mathrm{mV} \mathrm{s}^{-1}$ until the response was stable. Step $2: \ln$ order to study the oxygen evolution reaction kinetics, it is important to avoid any transient oxidation process such as the oxidation of $\mathrm{Ni}(\mathrm{OH})_{2}$ to $\mathrm{NiOOH}$. For this purpose, chronopotentiometric steps (CP steps) were performed at different fixed current densities $\left(j=0,5,10,25,50\right.$ and $\left.100 \mathrm{~mA} \mathrm{~cm}^{-2}\right)$ for 5 minutes each with stirring. In some cases, a stable potential was not obtained after 5 minutes, so the CP steps were extended by 5 additional minutes. The $\left(j, E_{j}\right)$ data points were collected. The overpotential at a given current density $j\left(\eta_{j}\right)$ was calculated according to the following equation $\eta_{j}=\mathrm{E}_{j}-1.23$ with $\mathrm{E}_{j}$ the potential measured at the current density $j$, in $\mathrm{V}$ vs $\mathrm{RHE}$. The $\left(j, \eta_{j}\right)$ points were plotted in a $j-\eta$ graph. Tafel slopes were obtained by plotting $\eta$ against $\log j$. The linear fit of these plots: $\eta=a+b \cdot \log j$ gives the Tafel slope b. Step 3 : The short-term stability of the different samples in each electrolyte was 
tested by running electrolysis at a fixed current density $j$ of $50 \mathrm{~mA} \mathrm{~cm}{ }^{-2}$ for 30 minutes under stirring. The $\mathrm{pH}$ of the electrolyte in the anode compartment did not change during electrolysis.

Electrochemically active surface area evaluation The double-layer capacitance $C_{D L}$ values were determined electrochemically in an aqueous solution of $1 \mathrm{M} \mathrm{KOH}$. All measurements were conducted in the voltage range $+0.95-+1.05 \mathrm{~V}$ vs RHE as it is a non-Faradaic region for most of the studied samples as well as for the NF support. An exception was made for $\mathrm{Cu}-\mathrm{O}$, which shows a Faradaic process in this region and therefore the double layer capacitance was measured in the range $+0.66-$ $+0.76 \mathrm{~V}$ vs RHE for this sample. The difference between the anodic and cathodic charging currents $\Delta j$ was obtained from CV scans at different scan rates (from 20 to $600 \mathrm{mV} \mathrm{s}^{-1}$ ). The double-layer capacitance is given by $\Delta j / 2=v \cdot C_{D L}$ where $v$ is the scan rate. Electrochemically active surface areas (ECSAs) could theoretically be obtained using the relation ECSA $=C_{D L} / C_{S}$ where $C_{S}$ is the specific capacitance of the sample, which corresponds to the capacitance of an atomically smooth planar surface of the same material per unit area under identical electrolyte conditions. However, it is impossible to determine reliable values of $C_{S}$ for each sample. Note that in previous studies, for determination of ECSAs of various OER catalysts, the same value of $C_{\mathrm{s}}=0.040 \mathrm{mF} \mathrm{cm}^{-2}$ in $1 \mathrm{M} \mathrm{NaOH}$, based on typical reported values for metallic surfaces, was applied. ${ }^{27,28}$ However, one should be aware that this only gives an estimation of the ECSAs since the $C_{S}$ value varies significantly from one material to another. $^{27}$

Pre-OER oxidation wave analysis The charge passed during the pre-OER oxidative process was evaluated. Each sample was maintained at a reducing potential ( $+0.4 \mathrm{~V}$ vs $\mathrm{RHE})$ for 20 minutes in order to start from a fully reduced catalyst. Linear sweep voltammetry scans were recorded from +0.40 to $+1.62 \mathrm{~V}$ vs RHE, with scan rates ranging from 1 to $100 \mathrm{mV} / \mathrm{s}$. Between each scan rate, the sample was kept at $+0.4 \mathrm{~V}$ for 5 minutes. An exponential background was subtracted from the $i=f(E)$ curve in order to deconvolute the contributions from the pre-OER redox process and the OER catalysis, which often occur in similar ranges of potential. The charge $Q$ passed during the pre-OER process was calculated from the integration of the 
oxidative wave: $Q=(1 / \mathrm{U}) \cdot \int \mathrm{i}(\mathrm{E}) \mathrm{dE}$ with $\mathrm{u}$ the scan rate in $\mathrm{V} / \mathrm{s}$. The charge value was taken in a range where $Q$ is independent from the scan rate.

Metal content evaluation Each catalyst deposited on NF with a $1 \mathrm{~cm}^{2}$ geometric area was carefully removed from the support. Note that this operation was not possible in the case of $\mathrm{NiFe}-\mathrm{OOH}$ and CoV-O. The collected powders were dissolved in $65 \% \mathrm{HNO}_{3}$ at room temperature for 10 days in Teflon tubes. The solutions were diluted with $2 \% \mathrm{HNO}_{3}$ and analysed using inductively coupled plasma - mass spectroscopy (ICP-MS). The quantity of each metal in each catalyst was calculated. The total number of moles of metals $n_{\mathrm{M}}$ is the sum of the number of moles of each metal contained in the catalyst. For instance, in the case of NiMoFe-O, $n_{\mathrm{M}}=n_{\mathrm{Ni}}+n_{\mathrm{Mo}}+n_{\mathrm{Fe}}$. This assumes that all the metals are potentially OER active, which is only a rough estimation as the exact nature/composition of the active sites is unknown.

Stability test in flow conditions Stability measurements were performed in a 2-electrode electrochemical flow cell FLC-Standard purchased from Sphere Energy (Figure S1). The potential was measured using a leak free $\mathrm{Ag} / \mathrm{AgCl} / \mathrm{KCl}_{3.4 \mathrm{~m}}$ micro reference (Innovative instruments Ltd.). The gas produced in the anodic compartment was analysed by gas chromatography every 30 minutes using a SRI $8610 \mathrm{C}$ gas chromatograph equipped with a packed Molecular Sieve $5 \AA$ column for permanent separation. Argon (Linde 5.0) was used as carrier gas, the flow rate was regulated using a mass flow controller (Bronkhorst). A thermal conductivity detector (TCD) was used to quantify $\mathrm{O}_{2}$. The Faradaic efficiency $\left(\mathrm{FE}_{\mathrm{O}_{2}}\right)$ was calculated by dividing the measured amount of oxygen by the theoretical amount of $\mathrm{O}_{2}$ expected: $\mathrm{FE}_{\mathrm{O}_{2}}=\mathrm{n}_{\mathrm{O}_{2} \text {, measured }} / \mathrm{n}_{\mathrm{O}_{2} \text {,expected }}=\mathrm{n}_{\mathrm{O}_{2} \text {, measured }} \cdot 4 F / Q$ where $\mathrm{n}_{\mathrm{O}_{2} \text {, measured }}$ and $\mathrm{n}_{\mathrm{O}_{2} \text {,expected }}$ are the measured and expected amounts of $\mathrm{O}_{2}, Q$ the charge passed and $F$ the Faraday constant. An aliquot of anolyte was collected every hour. The aliquots collected were analysed via inductively coupled plasma - mass spectrometry (ICP-MS). 
Results Synthesis and characterisation of OER catalysts Nine catalysts were selected among the most active materials reported in the literature (Table 1 ). Cu-O and NiMoFe-O were cathodically electrodeposited on Ni foam (Figure $1 \mathrm{~A}$ ) from aqueous solutions of the metallic precursors. This simple method resulted in the formation of dendritic structures as shown by SEM analysis (Figure 1B, 1C, S2, S4). NiMoFe-O showed a particularly strong adhesion to the substrate because the presence of nickel in the as-deposited catalyst ensures a continuous interface with NF in terms of composition and thus a small lattice mismatch. EDX was used to confirm the elemental composition (Figure S3, S5). CoPi was anodically electrodeposited, which resulted in the slow formation of large dendrites with a poor adhesion to the NF support (Figure 1D, S6, S7) . FeCoW (Figure 1E, S8-A, S8B, S9) and CoV-OOH (Figure 1F, S10, S11) were synthesised from nanomaterial dispersions mixed with a Nafion ink and drop-cast and dried onto the surface of the support to form thick layers, which remained well attached to the support despite large cracks (Figures S8-B, S10-C). The major limitation of this method is the hydrophobicity of dry Nafion which promotes the formation of an air film trapped at the catalyst/electrolyte interface and limits their contact area. In order to reach a stable OER activity, these catalysts must be kept under oxidative conditions $\left(10 \mathrm{~min}\right.$ at $\left.5 \mathrm{~mA} \mathrm{~cm}{ }^{-2}\right)$ in an aqueous electrolyte in order for the Nafion's hydrophilic domains to swell and become predominant in the bulk. ${ }^{48} \mathrm{~A}$ modification in the nanostructure of FeCoW was observed after this process, revealing the formation of a thin layer structure (Figure S8-C, S8-D). We conclude that once the Nafion network is fully hydrated the metallic sites are exposed to the electrolyte. This allows dissolution/precipitation equilibria at the interface, resulting in the formation of highly nanostructured surfaces. NiFe-OOH (Figure 1G, S12, S13) was made through galvanic exchange of the Ni-based substrate with $\mathrm{Fe}^{3+}$ precursor, followed by the deposition 
of a bimetallic oxy-hydroxide. As the support is the only source of nickel, it is etched during the reaction. This ensures a very high adhesion of the catalyst on the support, but also slightly modifies the material by etching its $\mathrm{Ni}$ backbone. Nevertheless, this method is particularly interesting as it is very simple. NiFeSe and CoFeSe derived oxides were synthesised by a more complex three-step procedure

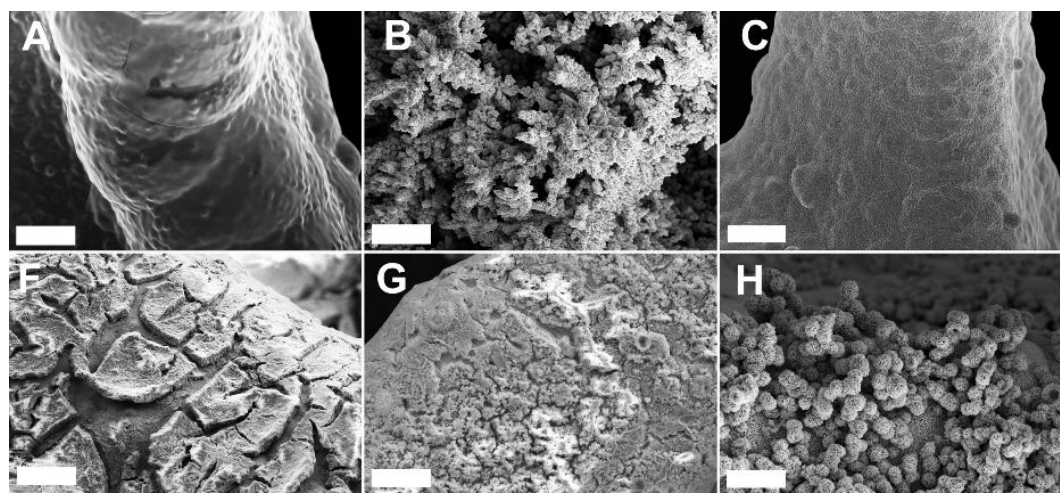

Figure 1 Scanning Electron Microscopy images of OER catalysts A the bare nickel foam, B Cu-O, C

NiMoFe-O, D CoPi, E FeCoW, F CoV-OOH, G NiFe-OOH, H NiFeSe, I CoFeSe, J CoV-O. Scale bar: 20 um. involving hydrothermal formation of layered double hydroxides (LDHs), followed by selenisation and subsequent reoxidation. The SEM images and EDX elemental analysis of the resulting NiFeSe-dO (Figure $1 \mathrm{H}, \mathrm{S} 14, \mathrm{~S} 15)$ and CoFeSe-dO (Figure $1 \mathrm{I}, \mathrm{S} 16, \mathrm{~S} 17)$ catalysts revealed the formation of very dense, thick and mechanically stable deposits at the surface of NF with fine nanostructures in the range of $30-100 \mathrm{~nm}$. However, the formation of a selenide involves hazardous synthesis steps and its reoxidation leads to toxic selenite waste products. CoV-O was synthesised by a simple one-step hydrothermal procedure involving the coprecipitation of $\mathrm{Co}$ and $\mathrm{V}$ in a mixed phase composed of a fine LDH nanostructure (Figure 1J, S18, S19) - this method is simple but results in a very low loading on NF.

It should be noted that the atomic compositions of the catalysts differed slightly from those reported in some cases. For example, in the case of NiMoFe-O (Figure S5), the use of NF as the support led to small modifications in the chemical composition of the film. The tungsten content in 
FeCoW is also lower than expected (Figure S9). Additionally, as the procedure for CoFeSe-dO synthesis could not be reproduced, it was modified in order to reach a Co:Fe ratio comparable to the Ni:Fe ratio in NiFeSe-dO (Figures S15, S17). In spite of the modifications we had to make in the procedures, the morphologies of the catalysts were comparable to the literature, as illustrated by the SEM images in Figures S14 and S16.

\section{Catalytic performance of OER catalysts}

9

Methodology for OER tests The catalytic activity of each material was measured under alkaline conditions, in an aqueous $1 \mathrm{M} \mathrm{KOH}$ electrolyte solution. Chronopotentiometric steps (CP steps) were performed at different fixed current densities $\left(j=0,5,10,25,50\right.$ and $\left.100 \mathrm{~mA} \mathrm{~cm}^{-2}\right)$ for 5 minutes each under stirring. This method is better than linear sweep voltammetry as it ensures that exclusively the oxygen evolution reaction response is measured and other contributions to the current are eliminated, such as the oxidation of $\mathrm{Ni}(\mathrm{OH})_{2}$ to $\mathrm{NiOOH}$ in Ni-containing materials. ${ }^{49}$ Furthermore, these $\mathrm{CP}$ steps give some information regarding the stability of the potential measured at different current densities on a short time scale. A possible drawback resides in some additional ohmic drop associated with the accumulation of oxygen bubbles at the surface of the electrode. ${ }^{16,50}$

The $j-\eta$ profiles and overpotentials at $j=10$ and $100 \mathrm{~mA} \mathrm{~cm}{ }^{-2}$ of the tested catalysts are displayed in Figure $2 \mathrm{~A}$ and Figure $2 \mathrm{~B}$ respectively (see Figure S20 for error bars), with the exact values of the overpotentials at $j=10 \mathrm{~mA} \mathrm{~cm}^{-2}$ given in Table S1. Additionally, the electrodes were held at a fixed current density $\left(j=50 \mathrm{~mA} \mathrm{~cm}{ }^{-2}\right)$ for 30 minutes in static conditions to verify that measured potentials were stable over a longer reaction time. In all cases this confirmed no clear degradative reactions (see Figure S21 for stability data). 
1 OER activities at $10 \mathrm{~mA} \mathrm{~cm}^{-2}$ The overpotential $\eta_{10}$ value is considered a figure of merit for OER

2 catalysts. All the catalysts characterised in this study have $\eta_{10}$ values between 211 and $347 \mathrm{mV}$, a

3 significant $136 \mathrm{mV}$ range (Figure 2, Table 2). The lowest overpotentials were obtained for NiFeSe-dO

$4 \quad\left(\eta_{10}=211 \mathrm{mV}\right)$ and CoFeSe-dO, $\left(\eta_{10}=212 \mathrm{mV}\right)$. The highest overpotentials were obtained for CoV-O

$5 \quad\left(\eta_{10}=331 \mathrm{mV}\right)$ and $\mathrm{Cu}-\mathrm{O}\left(\eta_{10}=347 \mathrm{mV}\right)$. Comparison with literature data is shown in Table S1.

A

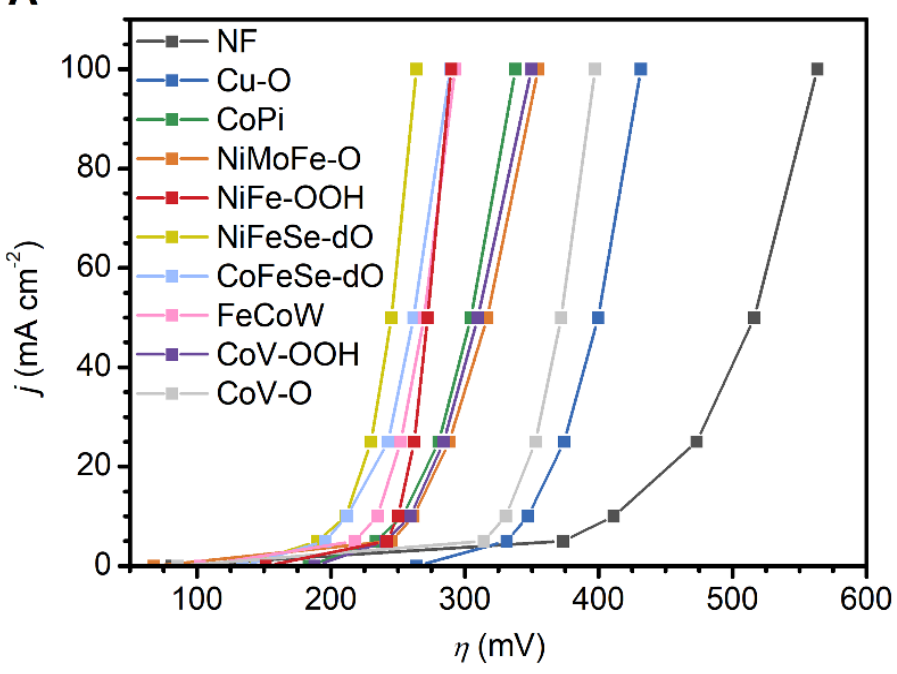

B

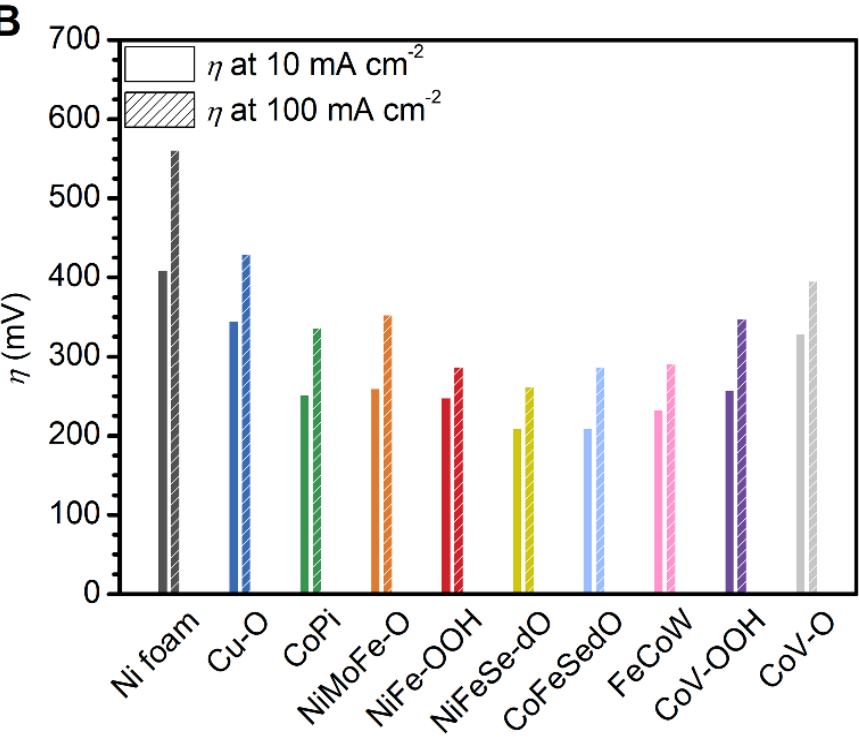

Figure 2 Electrocatalytic performance of OER catalysts in 1 M KOH A Current density versus overpotential (j-n) profiles obtained from CP steps analysis. B Overpotentials $\eta_{10}$ and $\eta_{100}$ obtained from CP steps. Data collected

\begin{tabular}{ccccccc} 
Catalyst & \multicolumn{2}{c}{ NF } & \multicolumn{2}{c}{ NiNF } & \multicolumn{2}{c}{$\Delta$ E (mV) } \\
\hline & $\eta_{10}$ & $\eta_{100}$ & $\eta_{10}$ & $\eta_{100}$ & $10 \mathrm{~mA} \mathrm{~cm}^{-2}$ & $100 \mathrm{~mA} \mathrm{~cm}^{-2}$ \\
\cline { 2 - 7 } Support & 411 & 563 & 331 & 434 & 80 & 129 \\
Cu-O & 347 & 432 & 328 & 418 & 19 & 13 \\
CoPi & 254 & 338 & 242 & 290 & 12 & 48 \\
NiMoFe-O & 262 & 355 & 257 & 314 & 5 & 41 \\
NiFe-OOH & 250 & 289 & 230 & 250 & 20 & 39 \\
NiFeSe-dO & 211 & 264 & 198 & 247 & 13 & 17 \\
CoFeSe-dO & 212 & 289 & 195 & 247 & 17 & 42 \\
FeCoW & 235 & 293 & 210 & 263 & 25 & 30 \\
CoV-OOH & 259 & 350 & 268 & 326 & -9 & 24 \\
CoV-O & 331 & 397 & 314 & 374 & 17 & 23
\end{tabular}

Table 2 Overpotentials obtained in $1 \mathrm{M} \mathrm{KOH}$ for our catalysts deposited either on NF or on NiNF $\eta_{10}$ and $\eta_{100}$ (in $\mathrm{mV}$ ) are the overpotentials at 10 and $100 \mathrm{~mA} \mathrm{~cm}^{-2}$ respectively, recorded under stirring at room temperature with a $85 \%$ iR-correction. $\triangle E$ represents the difference between NF and NiNF. 
OER activities at $100 \mathrm{~mA} \mathrm{~cm}$ $^{-2}$ Oxygen evolution must be carried out at higher current densities in order to meet the requirements for the electrochemical conversion and storage of renewable energy, such as solar-driven water splitting and $\mathrm{CO}_{2}$ reduction technologies. ${ }^{17,43-47,51}$ We therefore focus on the catalytic activities of the nine catalysts at $100 \mathrm{~mA} \mathrm{~cm}^{-2}$ (Figure 2, Table 2). While NF requires an overpotential as high as $563 \mathrm{mV}$, the nine catalysts enable an important drop in $\eta_{100}$ as compared to their support. Despite differences in the slopes, the trend in OER activity is the same at 10 and $100 \mathrm{~mA} \mathrm{~cm}^{-2}$. NiFeSe-dO remains at the head of the group with a low overpotential of $\eta_{100}=$ $264 \mathrm{mV}$, followed by CoFeSe-dO and NiFe-OOH with the same overpotential value of $289 \mathrm{mV}$, and by FeCoW with $\eta_{100}=293 \mathrm{mV}$. CoV-O $\left(\eta_{100}=397 \mathrm{mV}\right)$ and Cu-O $\left(\eta_{100}=432 \mathrm{mV}\right)$ show the lowest performances at all current densities.

Kinetics of OER catalysis The dependence of the OER kinetics on the applied potential for the catalysts is well illustrated through Tafel analysis (Figure S22 and Table S1). The fastest increase in current density upon potential increase occurs at the surface of NiFe-OOH, with a slope as low as 36 $m V$ dec $^{-1}$. NiFeSe-dO and FeCoW also showed low Tafel slope values of 55 and $56 \mathrm{mV} \mathrm{dec}{ }^{-1}$. The obtained values for the other catalysts ranged from 63 to $83 \mathrm{mV} \mathrm{dec}^{-1}$ (Figure S22). We systematically observed larger Tafel slopes with respect to reported ones, except for $\mathrm{NiFe}-\mathrm{OOH}$ (Table S1). This is likely because chronopotentiometric steps were used to measure Tafel slopes in place of LSV scans, which is the general methodology employed in the recent literature. Also, as a consequence of the CP steps, accumulation of $\mathrm{O}_{2}$ bubbles at the surface of the electrodes might contribute some extra resistance, especially at higher current densities, resulting in increased Tafel slope values. ${ }^{16,52,53}$ 

Comparing catalysts with different morphologies in terms of their intrinsic activities requires determination of the density of electrochemically active sites, a very important yet challenging analysis $^{54,55}$. Depending on parameters such as their nanostructure, porosity and lattice structure, catalysts can show very different interactions with the surrounding electrolyte. ${ }^{56-59}$ The density of electrochemically active and accessible sites can vary a lot from one catalyst to another. A range of techniques can be used to relate the total OER activity of a catalyst to the intrinsic activity of each active site. ${ }^{54,55}$

Two main methodologies are considered here in order to estimate the density of accessible active sites. The first is through determination of the electrochemically active surface area (ECSA) while the 
$\mathrm{mF} \mathrm{cm}{ }^{-2}$ in $1 \mathrm{M} \mathrm{NaOH}$, based on typical reported values for metallic surfaces, was applied. ${ }^{27,28}$ However, one should be aware that this only gives an estimation of the ECSAs since the $C_{\mathrm{S}}$ value varies significantly from one material to another. ${ }^{27}$ In our case, $C_{D L}$ were measured by cyclic voltammetry in the range $+0.95-+1.05 \mathrm{~V}$ vs $\mathrm{RHE}$, as it is a non-faradaic region for all our catalysts (except Cu-O which was characterised between +0.66 and $+0.76 \mathrm{~V}$ vs RHE). However, OER catalysts can show potential-dependent conductivity variations, therefore inaccuracies can arise from lower conductivities in potential regions prior to water oxidation. ${ }^{54,55,67}$

Here we report the $C_{\mathrm{DL}}$ values for the nine materials and for the NF support, measured in $1 \mathrm{M} \mathrm{KOH}$ using electrodes with $1 \mathrm{~cm}^{2}$ geometric areas (Figure 3A, S23). We indeed observed large differences: some of the studied catalysts have $C_{\mathrm{DL}}$ values in the range of $1 \mathrm{mF}$, slightly larger than that of the $\mathrm{Ni}$ foam (0.9 mF), while CoFeSe-dO, NiMoFe-O and NiFeSe-dO have much larger $C_{D L}$ values of 3.65, 2.40 and $2.35 \mathrm{mF}$, respectively. This result is in line with the observation of extremely fine nanostructures for these three catalysts (Figure $1 \mathrm{l}, 1 \mathrm{H}, 1 \mathrm{C}$ ).

Pre-OER oxidation wave analysis Most OER catalysts show redox features at potentials below the OER onset. These processes are generally attributed to the oxidation of the metal sites. For instance, $\mathrm{Ni}$ hydroxide oxidizes to $\mathrm{Ni}^{\text {III }}$ oxyhydroxide prior to OER catalysis. ${ }^{54,65,66}$ The amount of charge (Q) necessary to oxidize all the electrochemically active metal sites in a catalyst can be estimated through integration of the oxidation wave. $Q$ is directly proportional to the number of electrochemically accessible active sites (N) in the material according to the relation $\mathrm{N}=Q /\left(n_{e} \cdot Q_{e}\right)$, where $n_{e}$ is the number of electrons of the oxidation process observed and $Q_{e}$ is the charge of an electron. It is generally assumed that this oxidation is a one electron process, although this is a very strong assumption because the initial state of the catalyst is likely to be a mixed-valence state, with delocalized energy bands. ${ }^{68}$ Therefore, electron transfers are 
more complex than isolated one-electron transfers - here we chose to compare $Q$ values without assuming an arbitrary value for $n_{e}$. However, one should be aware that $Q$ can account for bulk sites which might not participate in the OER reaction, especially since recent studies have shown the prominent role of surface sites in OER catalysis. ${ }^{70,72}$ Another limitation of this method comes from the superposition of the oxidation wave and the OER onset in some cases - the deconvolution of these two contributions can be challenging. The simultaneous OER wave can also block a fraction of the active sites due to the formation of $\mathrm{O}_{2}$ bubbles and interference from mass transport limitations at high current density.

The estimated oxidation charges $Q$ for each of our nine catalysts with $1 \mathrm{~cm}^{2}$ geometric areas are displayed in Figure 3A. Most catalysts have $Q$ values comprised between 200 and $480 \mathrm{mC}$, except NiMoFe-O, NiFeSe-dO and CoFeSe-dO which stand out once again with values as high as 600,800 and 980 $\mathrm{mC}$, respectively. NiFe-OOH has a surprisingly low value of $60 \mathrm{mC}$. Despite some discrepancies (e.g. Cu-O and $\mathrm{NiFe}-\mathrm{OOH}$ ), it is interesting to observe that the $C_{D L}$ values correspond quite well with the obtained $Q$ values. Among the nine catalysts, CoFeSe-dO shows the highest density of active sites, followed by NiFeSe-dO and NiMoFe-O.

Intrinsic Activity The current densities measured for each catalyst at a fixed overpotential of $250 \mathrm{mV}$ are displayed in Figure 3B. We observe that NiFeSe-dO has the highest activity, followed by CoFeSe-dO, FeCoW and $\mathrm{NiFeOOH}$. The correlation of these data with those in Figure $3 \mathrm{~A}$ provides the following conclusions. First, NiFeSe-dO has a much higher intrinsic activity than CoFeSe-dO, since the current density is significantly higher than that of CoFeSe-dO, despite exhibiting a lower density of active sites. Therefore, NiFeSe-dO is the most active catalyst thanks to a combination of high density of active sites and high intrinsic activity of these sites, while the high activity of CoFeSe-dO is mainly due to the large density of active sites. Second, $\mathrm{FeCOW}$ and NiFe-OOH display quite high activities but have a low density 

relatively high intrinsic activities.

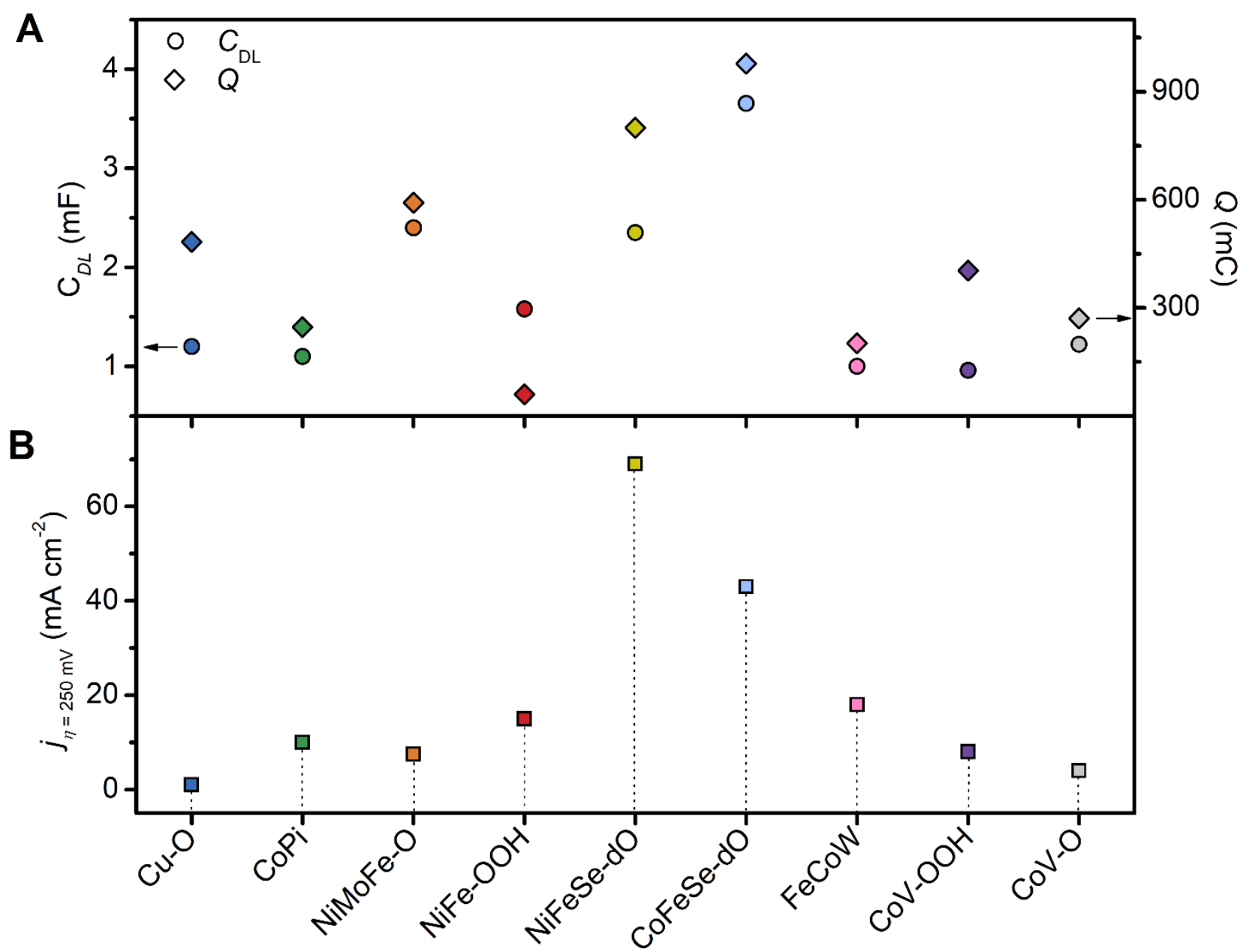

Figure 3 Characterisation of the electrochemically active sites of OER catalysts A Dots: double layer capacitances $C_{D L}$ obtained from plots of capacitive currents against the scan rate (Figure S23). The measurements were performed in $1 \mathrm{M} \mathrm{KOH}$, in the range $+0.95 \mathrm{~V}-+1.05 \mathrm{~V}$ vs RHE, which is a non-Faradaic region for most of these catalysts. For $\mathrm{Cu}-\mathrm{O}$ this potential range is a Faradaic region, so we performed the measure in a different range: $0.66-+0.76 \mathrm{~V}$ vs RHE, thus these values must be taken with care. Diamonds: charge passed during the pre-OER oxidation process, evaluated from the integration of $I=f(t)$ at different scan The evaluation of the density of active sites of metal-based (oxy)hydroxide materials is a highly challenging task. The two methods used here both suffer from limitations. Estimating ECSA using $C_{D L}$ 
measurements requires the use of specific capacitances and strongly depends on the conductivity of the material, which can vary upon application of a potential. The integration of pre-OER oxidation peaks relies on the assumption that single electron transfer steps operate, and calculation is often complicated by the deconvolution of OER catalytic wave. We show a correlation between the results of these two methods and stress the need to cross-check data using two complementary techniques. ${ }^{54,66}$ This should be considered as a best-practice protocol for researchers aiming to evaluate intrinsic catalytic activities.

Metals molar activity The evaluation of the total number of moles of metal atoms $\left(n_{M}\right)$ in a catalyst on a 1 $\mathrm{cm}^{2}$ geometric area electrode can provide access to another useful information, namely the mass activity or molar activity. A high molar activity effectively translates into a lower cost, as a lower number of metal atoms are required to perform OER catalysis at a given overpotential.

A $n_{\mathrm{M}}$ value can be obtained through dissolution of the catalyst layer and analysis using ICP-MS. The metal content of the nine catalysts are displayed in Figure S24-A. We observe that NiFeSe-dO and CoFeSe-dO have the lowest metal content whereas FeCoW has the highest. The metals molar activity was calculated by dividing the current density at $\eta=250 \mathrm{mV}$ by the metal content of each catalyst (Figure S24-B). The data clearly show that not only NiFeSe-dO and CoFeSe-dO display the largest current densities but they do it with the lowest amount of metals, therefore they show extremely high metal molar activities (520 and $634 \mathrm{~mA} \mathrm{~cm}^{-2} \mathrm{mmol}^{-1}$, respectively). In contrast, all other catalysts have much lower molar activities, in the $20-50 \mathrm{~mA} \mathrm{~cm}^{-2} \mathrm{mmol}^{-1}$ range. In the case of FeCoW, while the number of metals is high, only a small fraction is involved in the OER. Consequently, an improved exposition of the metal sites to the electrolyte might be key in the enhancement of the OER activity of this catalyst. 
Hierarchical structuration of the Ni foam support

5

A novel support with an increased surface area The NF support used in this study benefits from a relatively high ECSA of approximately $15 \mathrm{~cm}^{2} \mathrm{~cm}_{\text {geo }}{ }^{-2}$ (per geometric square centimetre), estimated using $C_{\mathrm{DL}}$ measurement for NF and $C_{\mathrm{S}}$ measurement using a Ni plate electrode (Figure S25). In order to further increase the surface area of this support, we used a straightforward method for electrodeposition of nickel dendrites on NF. ${ }^{69}$ The deposition of the metallic branched structures in the presence of protons at very high current density generates $\mathrm{H}_{2}$ bubbles at the surface of the electrode, creating a porous dendritic morphology (Figure 4A, S26). As a result of this increased structuration, the double layer capacitance of $\mathrm{NF}$ was greatly increased from $0.9 \mathrm{mF}$ to $4.9 \mathrm{mF}$ (Figure S27), leading to an estimated surface area of approximately $82 \mathrm{~cm}^{2} \mathrm{~cm}_{\text {geo }}{ }^{-2}$.
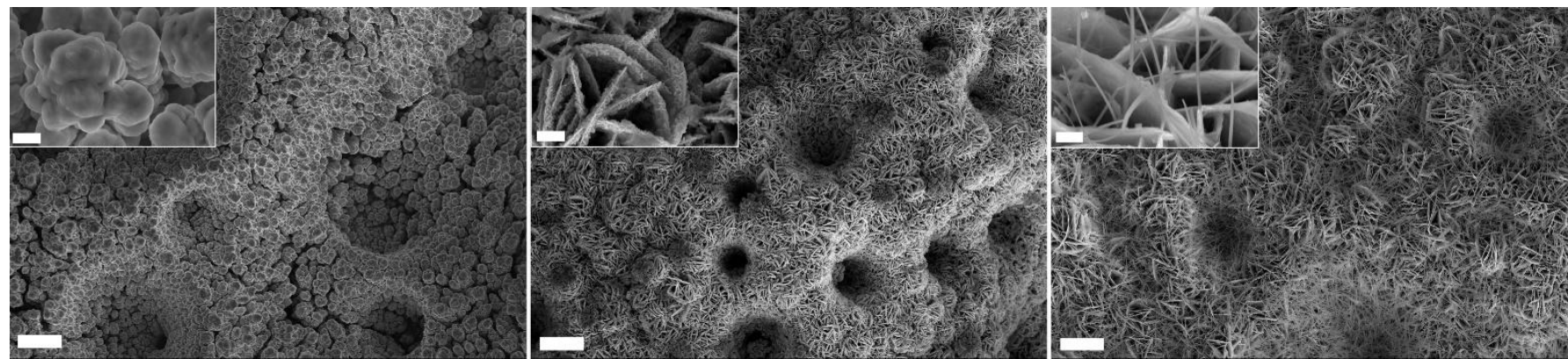

Figure 4 SEM characterisation of the NiNF support and its impact on the porosity of NiFeSe and CoFeSe A

The dendritic Ni foam NiNF, B NiFeSe deposited on the dendritic NiNF support and C CoFeSe deposited on

NiNF as a novel support leading to improved OER catalysts In line with our prior conclusion regarding the benefit of a higher surface area and a larger density of accessible active sites for OER activity, a significantly higher activity was observed for this dendritic nickel foam (NiNF) compared to NF. A 80 $\mathrm{mV}$ decrease of its $\eta_{10}$ value $\left(\eta_{10 \mid \text { NiNF }}=331 \mathrm{mV}\right)$, and a $129 \mathrm{mV}$ decrease of its $\eta_{100}$ value $\left(\eta_{100 \mid \text { NiNF }}=\right.$ 
$434 \mathrm{mV}$ ) were measured. This marked increase in activity makes NiNF a highly interesting anodic support material for electrolytic cells. NiNF was used as a new support for the deposition of the OER catalysts described above. We first detail the results obtained with the most active catalysts, namely NiFeSe-dO and CoFeSe-dO, deposited on NiNF (Figure 4B, 4C). From the SEM images, it appears that the nanostructure of NiFeSe-dO and CoFeSe-dO was maintained on this support, resulting in a hierarchical porous structure composed of three levels of porosities: the large pores of the nickel foam $(\approx 500 \mu \mathrm{m})$, the pores formed by the nickel dendrites $(\approx 1-10 \mu \mathrm{m})$ and the meso-/macropores resulting from the layered structure of the catalysts $\left(\approx 30-100 \mathrm{~nm}\right.$ ) (Figure 4B, 4C). The $C_{\mathrm{DL}}$ value of NiFeSe-dO on NiNF was a factor of four greater than on the NF, reaching a high $C_{\mathrm{DL}}$ value of $9.6 \mathrm{mF}$ (Figure S27-A). The $C_{\mathrm{DL}}$ value for CoFeSe-dO was roughly three times higher, giving an extremely large $C_{\mathrm{DL}}$ value of 10.3 $\mathrm{mF}$ (Figure S27-B). Therefore, the use of this highly porous support enables a significant increase in the density of accessible active sites. To determine how the increase in $C_{D L}$ impacted the catalytic activity, the NiNF-deposited catalysts were evaluated using our standard electrochemical characterisation procedure detailed in the previous sections (Figure 5). For both catalysts, the $\eta_{10}$ and $\eta_{100}$ values decreased upon substitution of NF with NiNF. For NiFeSe-dO, replacing NF with NiNF decreased $\eta_{10}$ from $211 \mathrm{mV}$ to $198 \mathrm{mV}$ and $\eta_{100}$ from $264 \mathrm{mV}$ to $247 \mathrm{mV}$ (Figure $5 \mathrm{~A}$ ). With CoFeSe-dO, the improvement was even more significant with $\eta_{10}$ decreasing from $212 \mathrm{mV}$ to $195 \mathrm{mV}$ and $\eta_{100}$ decreasing from $289 \mathrm{mV}$ to $247 \mathrm{mV}$ (Figure 5B). This represents a substantial improvement in performance of these OER catalysts (Table 2). The $\eta_{10}$ obtained for CoFeSe-dO and NiFeSe-dO are among the lowest value reported in the literature so far. The Tafel slope decreased from 55 to 54 $\mathrm{mV} \mathrm{dec}{ }^{-1}$ in the case of NiFeSe-dO, and from 72 to $63 \mathrm{mV} \mathrm{dec}^{-1}$ in the case of CoFeSe-dO (see Figures S28, S29 and Table 3). 

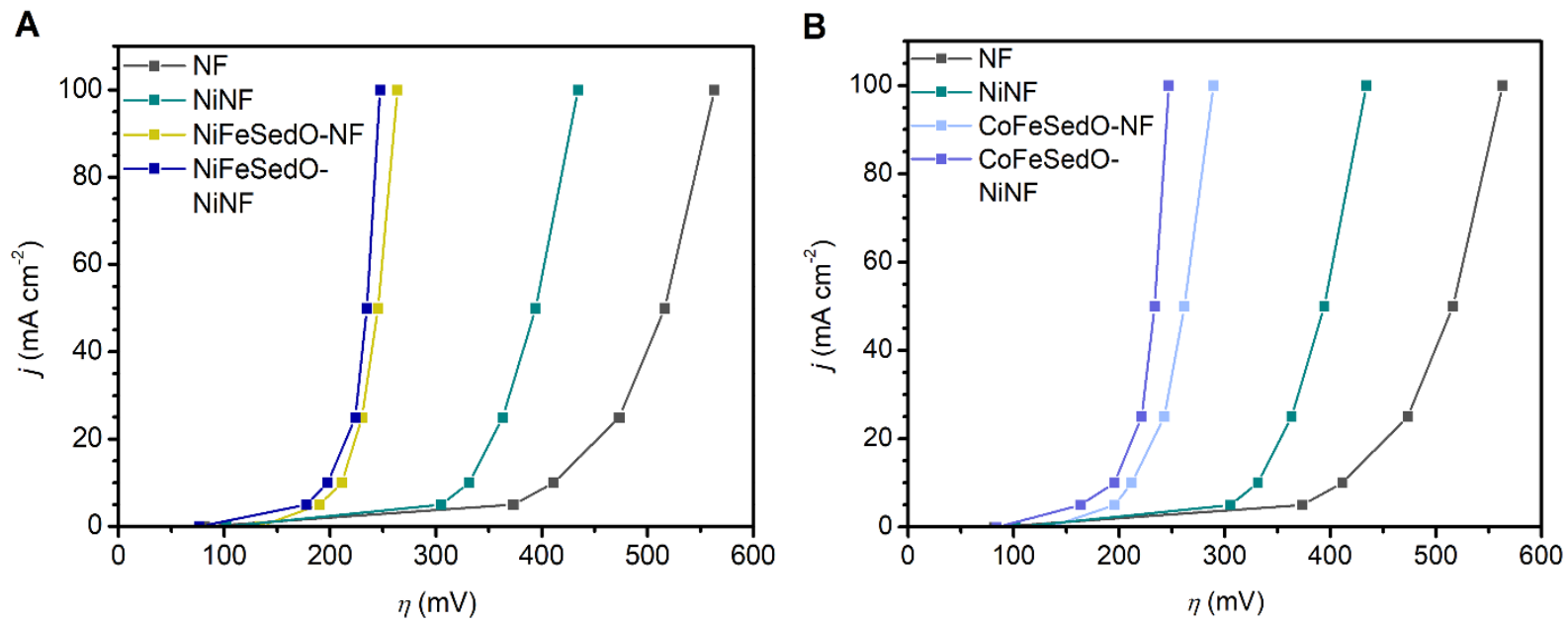

Figure 5 Impact of the NiNF support on the catalytic activity of NiFeSe-dO and CoFeSe-dO $j-n$ profiles obtained by recording chronopotentiometric steps in $1 \mathrm{M} \mathrm{KOH}$, under stirring at room temperature, using a $85 \%$ iR-correction. A NiFeSedO on NF and NiNF supports. B CoFeSe-dO on NF

For the other catalysts, we also observed a large increase in the $C_{\mathrm{DL}}$ by shifting from NF to NiNF as the support (Figures S30, S31). In all cases, apart from FeCoW, the $C_{\mathrm{DL}}$ values increased by a factor between 2.4 and 5, reflecting the improvement in specific surface area provided by NiNF. FeCoW shows a unique 9.7-fold increase in its $C_{\mathrm{DL}}$, larger than that of the support itself (Figure S31). This is likely due to an altered morphology of this catalyst when moving from NF to NiNF, leading to an increased density of accessible active sites. Additionally, a decrease of the overpotentials $\eta_{10}$ (by 5 to $80 \mathrm{mV}$ ) and $\eta_{100}$ (by 13 to $129 \mathrm{mV}$ ) (Figures S32-S38 and Table 2) and a decrease of the Tafel slopes (by 1 to $30 \mathrm{mV} \mathrm{dec}{ }^{-1}$, except for Cu-O) (Figures S32-S38 and Table 3) was observed. The gain of 20 to $30 \mathrm{mV} \mathrm{dec}{ }^{-1}$ in most cases is significant as it induces a large decrease in the overpotential at high current densities. In particular, NiFe-OOH displays a remarkably low Tafel slope of $20 \mathrm{mV} \mathrm{dec}{ }^{-1}$ when deposited on NiNF. 


\begin{tabular}{cccc} 
Catalyst & $\begin{array}{c}b^{N t} \\
\left(\mathrm{mV} \mathrm{dec}^{-1}\right)\end{array}$ & $\begin{array}{c}b^{\text {NIN }} \\
\left(\mathrm{mV} \mathrm{dec}^{-1}\right)\end{array}$ & $\begin{array}{c}\Delta b \\
\left(\mathrm{mV} \mathrm{dec}^{-1}\right)\end{array}$ \\
\hline Support & 147 & 97 & 50 \\
Cu-O & 76 & 83 & -7 \\
CoPi & 78 & 48 & 30 \\
NiMoFe-O & 83 & 53 & 30 \\
NiFe-OOH & 36 & 20 & 16 \\
NiFeSe-dO & 55 & 54 & 1 \\
CoFeSe-dO & 72 & 63 & 9 \\
FeCoW & 56 & 53 & 3 \\
CoV-OOH & 81 & 56 & 25 \\
CoV-O & 63 & 57 & 6
\end{tabular}

Table 3 Tafel slopes obtained for our catalysts deposited either on NF or on NiNF Tafel slopes $b$ (in $\mathrm{mV} \mathrm{dec}^{-1}$ ) were measured in $1 \mathrm{M} \mathrm{KOH}$, under stirring at room temperature using a $85 \% \mathrm{iR}$ correction. The corresponding change in $b(\Delta b)$ arises from the use of the dendritic NiNF support.

\section{Study of the stability of the materials in flow conditions}

Water splitting flow experiment Stable operation under continuous flow conditions at high current density is an important property of OER systems. We designed a water splitting experiment under flow conditions (Figure S1), in order to test our best catalysts (NiFeSedO-NiNF and CoFeSedO-NiNF) under conditions closer to industrial applications. The catalyst was loaded in a two-compartment cell separated by a Nafion ${ }^{\circledast}$ membrane with a platinum mesh-based cathode. The anolyte and catholyte were $1 \mathrm{M} \mathrm{KOH}$ aqueous solutions. These solutions were recirculated in each compartment from electrolyte containers. A current density of $100 \mathrm{~mA} \mathrm{~cm}{ }^{-2}$ was applied for 8 hours and the potential response as well as $\mathrm{FE}_{\mathrm{O}_{2}}$ were measured over time. Online monitoring of the elements present in solution during electrolysis also allowed quantitative measurement of metal leaching from the catalysts.

Potential stability The flow experiment described above was performed using NF, NiFeSedO-NiNF (Figure 6A) and CoFeSedO-NiNF (Figure 6B) as the anodes. As illustrated by Figure 6, these two catalysts show extremely stable potentials over 8 hours of electrolysis at a high current density of $100 \mathrm{~mA} \mathrm{~cm}{ }^{-2}$. NF 
performs oxygen evolution at $1.96 \pm 0.06 \mathrm{~V}$ vs RHE. The initial increase in potential is attributed to the oxidation of nickel, which occurs at the surface of the foam but also reaches its subsurface during the first hour of electrolysis under such a high current density. Both NiFeSedO-NiNF and CoFeSedO-NiNF perform oxygen evolution at potentials as low as $1.58 \pm 0.02 \mathrm{~V}$ vs RHE, with outstanding stability, which represents a major improvement as compared to NF. The faradaic efficiency for $\mathrm{O}_{2}$ production $\left(\mathrm{FE}_{\mathrm{O}_{2}}\right.$ ) was evaluated by measuring the amount of oxygen produced at the anode (Figure 6). During the first hour of experiment, the headspace of the anolyte container was saturated in gas. After this equilibration period, $\mathrm{FE}_{\mathrm{O}_{2}}$ was very stable, with mean values of $98.0 \pm 1.5 \%, 97.8 \pm 3.0 \%$ and $98.5 \pm 1.9 \%$ for NF, NiFeSedONiNF and CoFeSedO-NiNF respectively. This confirms that oxygen evolution was the only process occurring at the surface of these catalysts.

Chemical stability The concentrations of $\mathrm{Ni}, \mathrm{Co}$ and $\mathrm{Fe}$ in the anolyte were measured every hour by ICPMS (Figure 6). In the case of NF, the Ni concentration is in the range 40-120 ppb. This concentration does not increase over time, which proves the very high stability of this support in $1 \mathrm{M} \mathrm{KOH}$ under a high current density. The concentration of Fe is comprised between 250 and $450 \mathrm{ppb}$ and is stable over time. This Fe content in a $\mathrm{KOH}$ electrolyte is common. ${ }^{71,73}$ In the case of NiFeSedO-NiNF, we observe a small increase in the Ni concentration at the beginning of the experiment, as it reaches $300 \mathrm{ppb}$. After this small increase, the concentration slowly stabilises at around $100 \mathrm{ppb}$, which corresponds to the background concentration measured in the case of NF. No additional Ni was dissolved over the course of the reaction (Figure 6A). As a result, NiFeSedO-NiNF is an extremely stable catalyst in these conditions. As expected, no Co was detected for NF or NiFeSedO-NiNF. In the case of CoFeSedO-NiNF, Ni and Co concentrations in the range $200-700$ ppb were measured (Figure 6B). This means that some Co and some $\mathrm{Ni}$ are dissolved from the surface of the catalyst at the beginning of the experiment, but do not accumulate in the solution, thus revealing the absence of continuous dissolution over the course of the electrolysis. The Fe concentrations remained constant for both catalysts and correspond to the background concentration measured with bare NF. In conclusion, these two catalysts have shown a high 

density of $100 \mathrm{~mA} \mathrm{~cm}{ }^{-2}$ and an electrolyte flow of $9 \mathrm{~mL} \mathrm{~min}^{-1}$
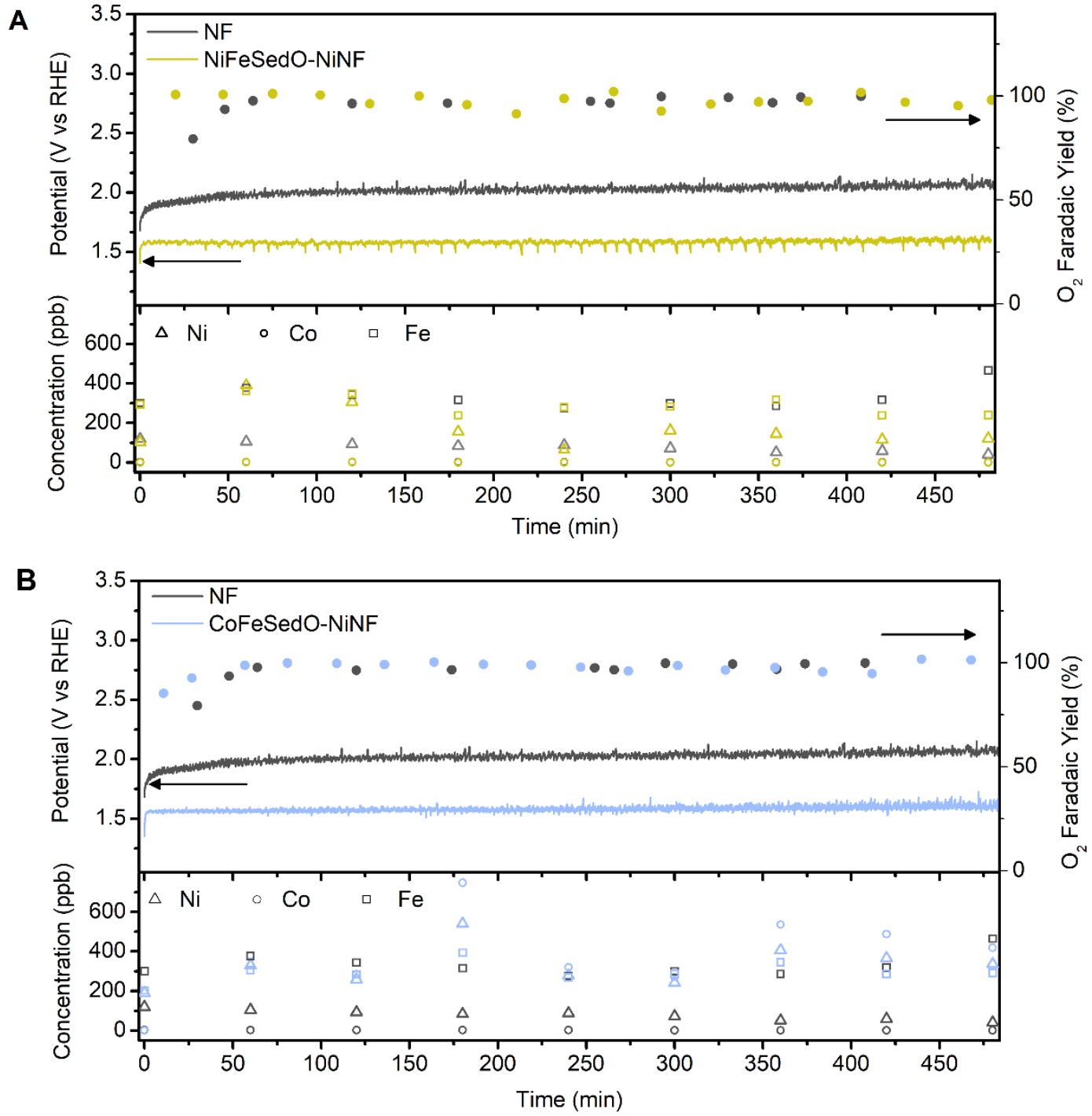

Figure 6 Stability of NiFeSedO-NiNF and CoFeSedO-NiNF in $1 \mathrm{M} \mathrm{KOH}$ at $100 \mathrm{~mA} \mathrm{~cm}$ A Potential measured during the test in flow conditions at $100 \mathrm{~mA} \mathrm{~cm}{ }^{-2}$ using NiFeSedO-NiNF as the anode. The potential is compared to the potential obtained with bare NF as the anode. A $1 \mathrm{M} \mathrm{KOH}$ solution was recirculated from a container in each compartment. The faradaic efficiency measured overtime for $\mathrm{O}_{2}$ production during the test in flow conditions using NF or NiFeSedO-NiNF is displayed on the right axis. The bottom plot shows Ni, Co and Fe concentrations (in $\mu \mathrm{g} \mathrm{L}^{-1}$ ) in the aqueous solution of $1 \mathrm{M} \mathrm{KOH}$ electrolyte flowing in the anode compartment during the stability test with NF (grey) or NiFeSedO-NiNF (yellow) used as the anode. B Potential, faradaic efficiency and ion concentrations data collected using CoFeSe-dO-NiNF as the anode (blue). The results are compared to those obtained with NF (grey). 


\section{Discussion}

For the first time, some of the most active OER catalysts reported during the last 4 years have been compared under identical reaction conditions after deposition on the same $\mathrm{Ni}$ foam support. For the reliability of the comparison, we used a standardised protocol to characterise the catalysts in terms of activity, density of active sites and stability. Specifically, $j-E$ curves were not obtained from LSVs, but instead from chronopotentiometric steps in $1 \mathrm{M} \mathrm{KOH}$ at different fixed current densities, up to 100 $\mathrm{mA} \mathrm{cm}{ }^{-2}$, to determine overpotentials. A range of complementary techniques was used in order to evaluate the density of accessible active sites of each anode. These procedures allowed for a reliable comparison of the catalysts.

The highest activities were observed for Se-doped bimetallic oxides, NiFeSe-dO and CoFeSe-dO, with remarkably low overpotentials of 211 and $212 \mathrm{mV}$ at $10 \mathrm{~mA} \mathrm{~cm}{ }^{-2}$, and 264 and $289 \mathrm{mV}$ at $100 \mathrm{~mA} \mathrm{~cm}^{-2}$ on a NF support, respectively. Their excellent overall OER activity is due to a combination of a high intrinsic activity and a high density of accessible active sites (Figure 3). The importance of selenium in precursor materials for OER has been previously discussed. ${ }^{37,38,74,75}$ During OER, Se is removed in solution, in the form of selenate and selenite ions, while oxygen atoms are incorporated. The metal selenide thus serves as a templating precursor to oxides/hydroxides which are the actual active species. Such Se-derived oxides display greater activity than oxides/hydroxides prepared by other methods. Furthermore, it is likely that the substitution of Se by $\mathrm{O}$ allows more active sites to be exposed, in line with the significantly higher densities of available active sites for the two Se-doped materials as compared to the other materials (Figure 3A). Moreover, in all cases, including here, significant amounts of Se atoms $(0.5-2 \mathrm{~mol} \%)$ are retained in the material after prolonged reaction. These atoms, which can be referred to as Se-doping, seem to improve the OER activity of the catalytic sites. A recent theoretical study indeed showed that Se-doping resulted in a substantial decrease of the energy barrier of the rate- 
determining step of the OER. ${ }^{74} \mathrm{NiFeSe}-\mathrm{dO}$ and CoFeSe-dO are unique, thanks to very high metal molar activities (Figure S24): these catalysts can perform OER at high current density while consuming few metal resources.

The combination of $\mathrm{Ni}$ and Fe sites provides the highest activity as has been observed in previous studies. ${ }^{11,75-78}$ In keeping with the very high performances measured for CoFeSe-dO, a similar beneficial association between $\mathrm{Co}$ and Fe is illustrated by the high catalytic activity of FeCoW, just below that of NiFeSe-dO and CoFeSe-dO, with a $\eta_{10}$ value of $235 \mathrm{mV}$ and a $\eta_{100}$ value of $293 \mathrm{mV}$ on the NF support. Analysis using NF indicates that FeCoW displays the highest intrinsic activity of the catalytic sites (Figure 3), but a very poor metal molar activity (Figure S24). Hence, further structuration leading to higher active surface areas of the deposit would be needed to improve the OER activity of FeCoW. Similar reasoning applies to $\mathrm{NiFeOOH}$.

Catalysts based on the association of Co and $\mathrm{V}$ are less active than the materials discussed above. CoV$\mathrm{OOH}$ shows a strikingly higher OER activity than CoV-O, which we attribute to the 50 -fold difference in mass loadings. Indeed, the hydrothermal synthesis procedure used for CoV-O led to the deposition of only $0.5 \mathrm{mg} \mathrm{cm}^{-2}$, whereas $25 \mathrm{mg} \mathrm{cm}^{-2}$ were drop-cast in the case of $\mathrm{CoV}-\mathrm{OOH}$. We do not discuss further the other catalysts, $\mathrm{CoPi}, \mathrm{Cu}-\mathrm{O}, \mathrm{NiMoFe}-\mathrm{O}$ as they show considerably lower activities than the most active catalysts discussed above.

We improved the structuration and the porosity of the electrodes through modification of the NF support by depositing dense dendritic and porous Ni structures. This was achieved using a fast and very simple electrodeposition procedure, where $\mathrm{H}_{2}$ bubbles act as templates for the formation of pores. A large increase in the double layer capacitance and greater OER performance than the untreated NF was observed. We tested this novel high-surface-area support, NiNF, with 9 different catalysts and in all cases we obtained increased double-layer capacitances, reflecting higher accessibility of active sites. We 
measured enhanced performances, as shown from decreased overpotentials and Tafel slopes. Specifically, the overpotentials of NiFeSe-dO and CoFeSe-dO further decreased to respectively 198 and $195 \mathrm{mV}$ at $10 \mathrm{~mA} \mathrm{~cm}^{-2}$ and to $247 \mathrm{mV}$ at $100 \mathrm{~mA} \mathrm{~cm}^{-2}$. These are amongst the lowest overpotentials ever reported in the literature.

NiFeSedO-NiNF and CoFeSedO-NiNF are the most active catalysts studied in this work. We decided to test their long-term stability. Most reports assess the stability of OER catalysts by applying a constant current and measuring the potential response. This method is relevant for preliminary tests because it enables fast analysis of extremely unstable catalysts. However, a constant potential response is not sufficient to claim that a catalyst is stable. Indeed, Kibsgarrd et al. ${ }^{79}$ underline that catalyst corrosion can impair its activity, but also improve it in certain cases because of factors including corrosion can lead to increased roughness. Moysiadou et al. ${ }^{80}$ draw attention to the observation that a catalyst can maintain stable macroscopic features during electrolysis, such as a stable potential, while experiencing a loss of mass due to a partial decomposition of its surface. As a result, activity monitoring does not provide a reliable measure of the overall stability of a catalyst. Transition metals at the surface of an oxygen evolution catalyst experience dissolution/redeposition equilibria during catalysis. ${ }^{56,81}$ Depending on the solubility of the metals considered, irreversible dissolution may occur. Moreover, the strong flow of electrolyte hitting the surface and the large amount of $\mathrm{O}_{2}$ bubbles generated can add to mechanical instability. For these reasons, leaching of metals from the surface of the catalyst is considered as a major route of decomposition, and a catalyst can only be claimed stable if leaching is negligible. This phenomenon can be detected by analysing the composition of the electrolyte after electrolysis by inductively coupled plasma - mass spectrometry (ICP-MS). ${ }^{82,83}$ Both electrochemical and compositional stabilities must be evaluated if one wants to prove the stability of a catalyst. This work proposes a methodology to perform stability evaluation by measuring galvanostatic features, $\mathrm{O}_{2}$ Faradaic efficiency and surface leaching all at once. This rigorous stability check, using a water splitting system, showed that NiFeSedO-NiNF and CoFeSedO-NiNF perform oxygen evolution in flow conditions, at a high current density of $100 \mathrm{~mA} \mathrm{~cm}{ }^{-2}$, 
with outstanding stability, be it on the macroscopic or on the microscopic scale. Constant potentials as low as $1.58 \mathrm{~V}$ vs RHE were measured at the anode. Extremely small amounts of transition metals were leached from the catalysts' surfaces, resulting in $\mathrm{Ni}, \mathrm{Co}$ and $\mathrm{Fe}$ concentrations generally comprised between $100-700 \mathrm{ppb}$ in the electrolyte. These results thus prove the excellent chemical and catalytic stability of the two catalysts. 


\section{Conclusion}

The benchmarking study presented here provides a reliable comparison between OER anodes comprised of catalysts deposited on porous supports. Nine of the most active and relevant precious-metal-free multimetallic OER catalysts were synthesised on nickel foams (NF) and compared in alkaline conditions (1 $\mathrm{M} \mathrm{KOH)} \mathrm{using} \mathrm{a} \mathrm{standardised} \mathrm{protocol.} \mathrm{The} \mathrm{overpotentials} \mathrm{at} 10 \mathrm{~mA} \mathrm{~cm}$ and $100 \mathrm{~mA} \mathrm{~cm}$ showed similar trends in OER activities. We identified NiFeSe-dO and CoFeSe-dO as the two best catalysts on NF, both showing $\eta_{10}$ values of $\approx 210 \mathrm{mV}$ and $\eta_{100}$ values of $264 \mathrm{mV}$ and $289 \mathrm{mV}$, respectively. We propose a protocol to assess the density of available active sites using complementary techniques (combining double layer capacitance measurements and pre-OER oxidation wave integration) and provide a qualitative comparison between intrinsic activities. NiFeSe-dO, in particular, stands out as a catalyst with high intrinsic activity, exhibiting higher currents than CoFeSe-dO despite displaying comparatively lower density of active sites. With insights from the relationship between available active sites and overall activity, we further enhanced the surface area of NF using an electrodeposition technique to texture the surface and form NiNF. OER catalysts displayed better performances (decreased overpotentials and Tafel slopes) when deposited on this new substrate, in the best case giving $\eta_{100}$ values of $247 \mathrm{mV}$ for CoFeSedO and NiFeSe-dO. Electrochemical and compositional stability of these catalysts were both evaluated. They perform OER catalysis in flow conditions at $100 \mathrm{~mA} \mathrm{~cm}^{-2}$ maintaining a constant potential without undergoing significant leaching. The information gained from this study not only enables fair and reliable comparison of the best reported OER catalysts on NF, but also highlights the important role of the support in oxygen evolution seeing as enhanced performance was observed after structuration of the NF to form NiNF. The integration of these improved NiNF-based catalysts in electrolytic cells can increase overall energy efficiency and enhance the viability of electrically driven energy conversion and storage. 
2 Declaration of interests We have no conflicts of interest to declare. Conceptualization A.P., and M.F.; Methodology A.P., C.E.C., D.K., H.N.T., and M.F.; Investigation A.P., C.E.C., and D.K.; Writing - Original Draft A.P., and M.F.; Writing - Review \& Editing A.P., C.E.C., D.K., H.N.T., M.S. and M.F.; Funding Acquisition, M.S. and M.F.; Supervision C.E.C., and M.F.. Acknowledgments This work was supported financially by funding from TOTAL S.A. Parts of this work were supported by IPGP multidisciplinary program PARI, and by Paris-IdF region SESAME Grant no. 12015908. 
4 1. Kim, J.H., Hansora, D., Sharma, P., Jang, J.-W., and Lee, J.S. (2019). Toward practical solar hydrogen production - an artificial photosynthetic leaf-to-farm challenge. Chem. Soc. Rev. 48, 1908-1971.

2. Ager, J.W., Shaner, M.R., Walczak, K.A., Sharp, I.D., and Ardo, S. (2015). Experimental demonstrations of spontaneous, solar-driven photoelectrochemical water splitting. Energy Environ. Sci. 8, 2811-2824.

3. Abbasi, R., Setzler, B.P., Lin, S., Wang, J., Zhao, Y., Xu, H., Pivovar, B., Tian, B., Chen, X., Wu, G., et al. (2019). A Roadmap to Low-Cost Hydrogen with Hydroxide Exchange Membrane Electrolyzers. Adv. Mater. 31, 1805876.

4. Vincent, I., and Bessarabov, D. (2018). Low cost hydrogen production by anion exchange membrane electrolysis: A review. Renew. Sustain. Energy Rev. 81, 1690-1704.

5. Barber, J., and Tran, P.D. (2013). From natural to artificial photosynthesis. J. R. Soc. Interface 10, 20120984.

6. Kalamaras, E., Maroto-Valer, M.M., Shao, M., Xuan, J., and Wang, H. (2018). Solar carbon fuel via photoelectrochemistry. Catal. Today $317,56-75$.

7. Gabardo, C.M., O’Brien, C.P., Edwards, J.P., McCallum, C., Xu, Y., Dinh, C.-T., Li, J., Sargent, E.H., and Sinton, D. (2019). Continuous Carbon Dioxide Electroreduction to Concentrated Multi-carbon Products Using a Membrane Electrode Assembly. Joule 3, 2777-2791.

8. Liang, S., Altaf, N., Huang, L., Gao, Y., and Wang, Q. (2020). Electrolytic cell design for electrochemical $\mathrm{CO}_{2}$ reduction. J. $\mathrm{CO} 2$ Util. 35, 90-105.

9. Creissen, C.E., and Fontecave, M. (2020). Solar-Driven Electrochemical $\mathrm{CO}_{2}$ Reduction with Heterogeneous Catalysts. Adv. Energy Mater., 2002652.

10. Schalenbach, M., Zeradjanin, A.R., Kasian, O., Cherevko, S., and Mayrhofer, K.J.J. (2018). A 
perspective on low-temperature water electrolysis - Challenges in alkaline and acidic technology. Int. J. Electrochem. Sci. 13, 1173-1226.

11. Song, F., Busch, M.M., Lassalle-Kaiser, B., Hsu, C.-S., Petkucheva, E., Bensimon, M., Chen, H.M., Corminboeuf, C., and Hu, X. (2019). An Unconventional Iron Nickel Catalyst for the Oxygen Evolution Reaction. ACS Cent. Sci. 5, 558-568.

12. Suryanto, B.H.R., Wang, Y., Hocking, R.K., Adamson, W., and Zhao, C. (2019). Overall electrochemical splitting of water at the heterogeneous interface of nickel and iron oxide. Nat. Commun. 10, 5599

13. Long, X., Xiao, S., Wang, Z., Zheng, X., and Yang, S. (2015). Co intake mediated formation of ultrathin nanosheets of transition metal LDH-an advanced electrocatalyst for oxygen evolution reaction. Chem. Commun. 51, 1120-1123.

14. Zhou, Y., and Zeng, H.C. (2018). 3D Networks of CoFePi with Hierarchical Porosity for Effective OER Electrocatalysis. Small 14, 1704403.

15. Li, W., Li, F., Yang, H., Wu, X., Zhang, P., Shan, Y., and Sun, L. (2019). A bio-inspired coordination polymer as outstanding water oxidation catalyst via second coordination sphere engineering. Nat. Commun. 10, 5074.

16. Zeng, K., and Zhang, D. (2010). Recent progress in alkaline water electrolysis for hydrogen production and applications. Prog. Energy Combust. Sci. 36, 307-326.

17. Dinh, C.-T., Burdyny, T., Kibria, M.G., Seifitokaldani, A., Gabardo, C.M., García de Arquer, F.P., Kiani, A., Edwards, J.P., De Luna, P., Bushuyev, O.S., et al. (2018). $\mathrm{CO}_{2}$ electroreduction to ethylene via hydroxide-mediated copper catalysis at an abrupt interface. Science 360, 783787.

18. Xin, Y., Kan, X., Gan, L.-Y., and Zhang, Z. (2017). Heterogeneous Bimetallic Phosphide/Sulfide Nanocomposite for Efficient Solar-Energy-Driven Overall Water Splitting. ACS Nano 11, 1030310312.

19. Qiu, C., He, S., Wang, Y., Wang, Q., and Zhao, C. (2019). Interfacial Engineering $\mathrm{FeOOH} / \mathrm{CoO}$ 
Nanoneedle Array for Efficient Overall Water Splitting Driven by Solar Energy. Chem. - A Eur. J., chem. 201904352.

20. Suen, N.-T., Hung, S.-F., Quan, Q., Zhang, N., Xu, Y.-J., and Chen, H.M. (2017). Electrocatalysis for the oxygen evolution reaction: recent development and future perspectives. Chem. Soc. Rev. 46, 337-365.

21. Hunter, B.M., Gray, H.B., and Müller, A.M. (2016). Earth-Abundant Heterogeneous Water Oxidation Catalysts. Chem. Rev. 116, 14120-14136.

22. Song, F., Bai, L., Moysiadou, A., Lee, S., Hu, C., Liardet, L., and Hu, X. (2018). Transition Metal Oxides as Electrocatalysts for the Oxygen Evolution Reaction in Alkaline Solutions: An Application-Inspired Renaissance. J. Am. Chem. Soc. 140, 7748-7759.

23. Mohammed-Ibrahim, J. (2020). A review on NiFe-based electrocatalysts for efficient alkaline oxygen evolution reaction. J. Power Sources 448, 227375.

24. Benck, J.D., Pinaud, B.A., Gorlin, Y., and Jaramillo, T.F. (2014). Substrate Selection for Fundamental Studies of Electrocatalysts and Photoelectrodes: Inert Potential Windows in Acidic, Neutral, and Basic Electrolyte. PLoS One 9, e107942.

25. Chaudhari, N.K., Jin, H., Kim, B., and Lee, K. (2017). Nanostructured materials on 3D nickel foam as electrocatalysts for water splitting. Nanoscale 9, 12231-12247.

26. Trotochaud, L., Ranney, J.K., Williams, K.N., and Boettcher, S.W. (2012). Solution-Cast Metal Oxide Thin Film Electrocatalysts for Oxygen Evolution. J. Am. Chem. Soc. 134, 17253-17261.

27. McCrory, C.C.L., Jung, S., Peters, J.C., and Jaramillo, T.F. (2013). Benchmarking Heterogeneous Electrocatalysts for the Oxygen Evolution Reaction. J. Am. Chem. Soc. 135, 16977-16987.

28. McCrory, C.C.L., Jung, S., Ferrer, I.M., Chatman, S.M., Peters, J.C., and Jaramillo, T.F. (2015). Benchmarking Hydrogen Evolving Reaction and Oxygen Evolving Reaction Electrocatalysts for Solar Water Splitting Devices. J. Am. Chem. Soc. 137, 4347-4357.

29. Grdeń, M., Alsabet, M., and Jerkiewicz, G. (2012). Surface Science and Electrochemical Analysis of Nickel Foams. ACS Appl. Mater. Interfaces 4, 3012-3021. 
30. Hall, D.E. (1985). Alkaline Water Electrolysis Anode Materials. J. Electrochem. Soc. 132, 41C48C.

31. Hall, D.E. (1983). $\mathrm{Ni}(\mathrm{OH})_{2}$ - Impregnated Anodes for Alkaline Water Electrolysis. J. Electrochem. Soc. $130,317-321$.

32. Corrigan, D.A. (1987). The Catalysis of the Oxygen Evolution Reaction by Iron Impurities in Thin Film Nickel Oxide Electrodes. J. Electrochem. Soc. 134, 377-384.

33. Huan, T.N., Rousse, G., Zanna, S., Lucas, I.T., Xu, X., Menguy, N., Mougel, V., and Fontecave, M. (2017). A Dendritic Nanostructured Copper Oxide Electrocatalyst for the Oxygen Evolution Reaction. Angew. Chemie Int. Ed. 56, 4792-4796.

34. Esswein, A.J., Surendranath, Y., Reece, S.Y., and Nocera, D.G. (2011). Highly active cobalt phosphate and borate based oxygen evolving catalysts operating in neutral and natural waters. Energy Environ. Sci. 4, 499-504.

35. Jayalakshmi, M., Kim, W.-Y., Jung, K.-D., and Joo, O.-S. (2008). Electrochemical Characterization of Ni-Mo-Fe Composite Film in Alkali Solution. Int. J. Electrochem. Sci. 3, 908917.

36. Zhou, H., Yu, F., Zhu, Q., Sun, J., Qin, F., Yu, L., Bao, J., Yu, Y., Chen, S., and Ren, Z. (2018). Water splitting by electrolysis at high current densities under 1.6 volts. Energy Environ. Sci. 11, $2858-2864$.

37. Xu, X., Song, F., and Hu, X. (2016). A nickel iron diselenide-derived efficient oxygen-evolution catalyst. Nat. Commun. 7, 12324.

38. Zhang, J.-Y., Lv, L., Tian, Y., Li, Z., Ao, X., Lan, Y., Jiang, J., and Wang, C. (2017). Rational Design of Cobalt-Iron Selenides for Highly Efficient Electrochemical Water Oxidation. ACS Appl. Mater. Interfaces 9, 33833-33840.

39. Zhang, B., Zheng, X., Voznyy, O., Comin, R., Bajdich, M., Garcia-Melchor, M., Han, L., Xu, J., Liu, M., Zheng, L., et al. (2016). Homogeneously dispersed multimetal oxygen-evolving catalysts. Science $352,333-337$. 
40. Liu, J., Ji, Y., Nai, J., Niu, X., Luo, Y., Guo, L., and Yang, S. (2018). Ultrathin amorphous cobaltvanadium hydr(oxy)oxide catalysts for the oxygen evolution reaction. Energy Environ. Sci. 11, $1736-1741$

41. Liardet, L., and Hu, X. (2018). Amorphous Cobalt Vanadium Oxide as a Highly Active Electrocatalyst for Oxygen Evolution. ACS Catal. 8, 644-650.

42. Ma, W., Ma, R., Wang, C., Liang, J., Liu, X., Zhou, K., and Sasaki, T. (2015). A Superlattice of Alternately Stacked Ni-Fe Hydroxide Nanosheets and Graphene for Efficient Splitting of Water. ACS Nano 9, 1977-1984.

43. Jouny, M., Luc, W., and Jiao, F. (2018). General Techno-Economic Analysis of $\mathrm{CO}_{2}$ Electrolysis Systems. Ind. Eng. Chem. Res. 57, 2165-2177.

44. Lamaison, S., Wakerley, D., Blanchard, J., Montero, D., Rousse, G., Mercier, D., Marcus, P., Taverna, D., Giaume, D., Mougel, V., et al. (2020). High-Current-Density $\mathrm{CO}_{2}$-to-CO Electroreduction on Ag-Alloyed Zn Dendrites at Elevated Pressure. Joule 4, 395-406.

45. Jia, J., Seitz, L.C., Benck, J.D., Huo, Y., Chen, Y., Ng, J.W.D., Bilir, T., Harris, J.S., and Jaramillo, T.F. (2016). Solar water splitting by photovoltaic-electrolysis with a solar-to-hydrogen efficiency over 30\%. Nat. Commun. 7, 13237.

46. Nakamura, A., Ota, Y., Koike, K., Hidaka, Y., Nishioka, K., Sugiyama, M., and Fujii, K. (2015). A $24.4 \%$ solar to hydrogen energy conversion efficiency by combining concentrator photovoltaic modules and electrochemical cells. Appl. Phys. Express 8, 107101.

47. Khan, M.A., Al-Shankiti, I., Ziani, A., Wehbe, N., and Idriss, H. (2020). A Stable Integrated Photoelectrochemical Reactor for $\mathrm{H}_{2}$ Production from Water Attains a Solar-to-Hydrogen Efficiency of $18 \%$ at 15 Suns and $13 \%$ at 207 Suns. Angew. Chemie Int. Ed. 59, 14802-14808.

48. Zhao, Q., Majsztrik, P., and Benziger, J. (2011). Diffusion and Interfacial Transport of Water in Nafion. J. Phys. Chem. B 115, 2717-2727.

49. Hall, D.S., Bock, C., and MacDougall, B.R. (2013). The Electrochemistry of Metallic Nickel: Oxides, Hydroxides, Hydrides and Alkaline Hydrogen Evolution. J. Electrochem. Soc. 160, 
F235-F243.

50. Angulo, A., van der Linde, P., Gardeniers, H., Modestino, M., and Fernández Rivas, D. (2020). Influence of Bubbles on the Energy Conversion Efficiency of Electrochemical Reactors. Joule 4, $555-579$

51. De Luna, P., Hahn, C., Higgins, D., Jaffer, S.A., Jaramillo, T.F., and Sargent, E.H. (2019). What would it take for renewably powered electrosynthesis to displace petrochemical processes? Science 364 , eaav3506.

52. Sequeira, C.A.C., Santos, D.M.F., Šljukić, B., and Amaral, L. (2013). Physics of Electrolytic Gas Evolution. Brazilian J. Phys. 43, 199-208.

53. García-Osorio, D.A., Jaimes, R., Vazquez-Arenas, J., Lara, R.H., and Alvarez-Ramirez, J. (2017). The Kinetic Parameters of the Oxygen Evolution Reaction (OER) Calculated on Inactive Anodes via EIS Transfer Functions: • OH Formation. J. Electrochem. Soc. 164, E3321-E3328.

54. Stevens, M.B., Enman, L.J., Batchellor, A.S., Cosby, M.R., Vise, A.E., Trang, C.D.M., and Boettcher, S.W. (2017). Measurement Techniques for the Study of Thin Film Heterogeneous Water Oxidation Electrocatalysts. Chem. Mater. 29, 120-140.

55. Wei, C., Rao, R.R., Peng, J., Huang, B., Stephens, I.E.L., Risch, M., Xu, Z.J., and Shao-Horn, Y. (2019). Recommended Practices and Benchmark Activity for Hydrogen and Oxygen Electrocatalysis in Water Splitting and Fuel Cells. Adv. Mater. 31, 1806296.

56. Yang, C., Batuk, M., Jacquet, Q., Rousse, G., Yin, W., Zhang, L., Hadermann, J., Abakumov, A.M., Cibin, G., Chadwick, A., et al. (2018). Revealing pH-Dependent Activities and Surface Instabilities for Ni-Based Electrocatalysts during the Oxygen Evolution Reaction. ACS Energy Lett. 3, 2884-2890.

57. Zhang, R., Pearce, P.E., Duan, Y., Dubouis, N., Marchandier, T., and Grimaud, A. (2019). Importance of Water Structure and Catalyst-Electrolyte Interface on the Design of Water Splitting Catalysts. Chem. Mater. 31, 8248-8259.

58. Duan, Y., Dubouis, N., Huang, J., Dalla Corte, D.A., Pimenta, V., Xu, Z.J., and Grimaud, A. 
(2020). Revealing the Impact of Electrolyte Composition for Co-Based Water Oxidation Catalysts by the Study of Reaction Kinetics Parameters. ACS Catal. 10, 4160-4170.

59. Yang, C., Rousse, G., Louise Svane, K., Pearce, P.E., Abakumov, A.M., Deschamps, M., Cibin, G., Chadwick, A. V., Dalla Corte, D.A., Anton Hansen, H., et al. (2020). Cation insertion to break the activity/stability relationship for highly active oxygen evolution reaction catalyst. Nat. Commun. 11, 1378

60. Trasatti, S., and Petrii, O.A. (1992). Real surface area measurements in electrochemistry. J. Electroanal. Chem. 327, 353-376.

61. Burke, L.D., and O'Sullivan, E.J.M. (1981). Oxygen gas evolution on hydrous oxides - An example of three-dimensional electrocatalysis? J. Electroanal. Chem. Interfacial Electrochem. $117,155-160$.

62. Doyle, R.L., Godwin, I.J., Brandon, M.P., and Lyons, M.E.G. (2013). Redox and electrochemical water splitting catalytic properties of hydrated metal oxide modified electrodes. Phys. Chem. Chem. Phys. 15, 13737.

63. Klingan, K., Ringleb, F., Zaharieva, I., Heidkamp, J., Chernev, P., Gonzalez-Flores, D., Risch, M., Fischer, A., and Dau, H. (2014). Water Oxidation by Amorphous Cobalt-Based Oxides: Volume Activity and Proton Transfer to Electrolyte Bases. ChemSusChem 7, 1301-1310.

64. Lin, F., and Boettcher, S.W. (2014). Adaptive semiconductor/electrocatalyst junctions in watersplitting photoanodes. Nat. Mater. 13, 81-86.

65. Batchellor, A.S., and Boettcher, S.W. (2015). Pulse-Electrodeposited Ni-Fe (Oxy)hydroxide Oxygen Evolution Electrocatalysts with High Geometric and Intrinsic Activities at Large Mass Loadings. ACS Catal. 5, 6680-6689.

66. Wei, C., Sun, S., Mandler, D., Wang, X., Qiao, S.Z., and Xu, Z.J. (2019). Approaches for measuring the surface areas of metal oxide electrocatalysts for determining their intrinsic electrocatalytic activity. Chem. Soc. Rev.

67. Jung, S., McCrory, C.C.L., Ferrer, I.M., Peters, J.C., and Jaramillo, T.F. (2016). Benchmarking 
nanoparticulate metal oxide electrocatalysts for the alkaline water oxidation reaction. J. Mater. Chem. A 4, 3068-3076.

68. Grimaud, A., Hong, W.T., Shao-Horn, Y., and Tarascon, J.-M. (2016). Anionic redox processes for electrochemical devices. Nat. Mater. 15, 121-126.

69. Yang, F., Cheng, K., Xue, X., Yin, J., Wang, G., and Cao, D. (2013). Three-dimensional porous Ni film electrodeposited on Ni foam: High performance and low-cost catalytic electrode for $\mathrm{H}_{2} \mathrm{O}_{2}$ electrooxidation in $\mathrm{KOH}$ solution. Electrochim. Acta 107, 194-199.

70. Corby, S., Tecedor, M.G., Tengeler, S., Steinert, C., Moss, B., Mesa, C.A., Heiba, H.F., Wilson, A.A., Kaiser, B., Jaegermann, W., et al. (2020). Separating bulk and surface processes in NiOxelectrocatalysts for water oxidation. Sustain. Energy Fuels.

71. Smith, A.M., Trotochaud, L., Burke, M.S., and Boettcher, S.W. (2015). Contributions to activity enhancement via Fe incorporation in $\mathrm{Ni}$-(oxy)hydroxide/borate catalysts for near-neutral $\mathrm{pH}$ oxygen evolution. Chem. Commun. 51, 5261-5263.

72. Roy, C., Sebok, B., Scott, S.B., Fiordaliso, E.M., Sørensen, J.E., Bodin, A., Trimarco, D.B., Damsgaard, C.D., Vesborg, P.C.K., Hansen, O., et al. (2018). Impact of nanoparticle size and lattice oxygen on water oxidation on NiFeOxHy. Nat. Catal.

73. Spanos, I., Tesch, M.F., Yu, M., Tüysüz, H., Zhang, J., Feng, X., Müllen, K., Schlögl, R., and Mechler, A.K. (2019). Facile Protocol for Alkaline Electrolyte Purification and Its Influence on a $\mathrm{Ni-Co}$ Oxide Catalyst for the Oxygen Evolution Reaction. ACS Catal. 9, 8165-8170.

74. Niu, S., Jiang, W.-J., Wei, Z., Tang, T., Ma, J., Hu, J.-S., and Wan, L.-J. (2019). Se-Doping Activates $\mathrm{FeOOH}$ for Cost-Effective and Efficient Electrochemical Water Oxidation. J. Am. Chem. Soc. 141, 7005-7013.

75. Yu, L., Wu, L., McElhenny, B., Song, S., Luo, D., Zhang, F., Yu, Y., Chen, S., and Ren, Z. (2020). Ultrafast room-temperature synthesis of porous S-doped Ni/Fe (oxy)hydroxide electrodes for oxygen evolution catalysis in seawater splitting. Energy Environ. Sci.

76. Dionigi, F., Zeng, Z., Sinev, I., Merzdorf, T., Deshpande, S., Lopez, M.B., Kunze, S., Zegkinoglou, 
I., Sarodnik, H., Fan, D., et al. (2020). In-situ structure and catalytic mechanism of NiFe and CoFe layered double hydroxides during oxygen evolution. Nat. Commun. 11, 2522.

77. Zhou, Y., and López, N. (2020). The Role of Fe Species on $\mathrm{NiOOH}$ in Oxygen Evolution Reactions. ACS Catal. 10, 6254-6261.

78. Burke, M.S., Zou, S., Enman, L.J., Kellon, J.E., Gabor, C.A., Pledger, E., and Boettcher, S.W. (2015). Revised Oxygen Evolution Reaction Activity Trends for First-Row Transition-Metal (Oxy)hydroxides in Alkaline Media. J. Phys. Chem. Lett. 6, 3737-3742.

79. Kibsgaard, J., and Chorkendorff, I. (2019). Considerations for the scaling-up of water splitting catalysts. Nat. Energy 4, 430-433.

80. Moysiadou, A., and Hu, X. (2019). Stability profiles of transition metal oxides in the oxygen evolution reaction in alkaline medium. J. Mater. Chem. A 7, 25865.

81. Zhang, R., Dubouis, N., Ben Osman, M., Yin, W., Sougrati, M.T., Corte, D.A.D., Giaume, D., and Grimaud, A. (2019). A Dissolution/Precipitation Equilibrium on the Surface of Iridium-Based Perovskites Controls Their Activity as Oxygen Evolution Reaction Catalysts in Acidic Media. Angew. Chemie Int. Ed. 58, 4571-4575.

82. Geiger, S., Kasian, O., Ledendecker, M., Pizzutilo, E., Mingers, A.M., Fu, W.T., Diaz-Morales, O., Li, Z., Oellers, T., Fruchter, L., et al. (2018). The stability number as a metric for electrocatalyst stability benchmarking. Nat. Catal. 1, 508-515.

83. Frydendal, R., Paoli, E.A., Knudsen, B.P., Wickman, B., Malacrida, P., Stephens, I.E.L., and Chorkendorff, I. (2014). Benchmarking the Stability of Oxygen Evolution Reaction Catalysts: The Importance of Monitoring Mass Losses. ChemElectroChem 1, 2075-2081. 